\title{
NeuroD Factors Regulate Cell Fate and Neurite Stratification in the Developing Retina
}

\author{
Timothy J. Cherry, ${ }^{1}$ Sui Wang, ${ }^{1,2}$ Ingo Bormuth, ${ }^{3,4}$ Markus Schwab, ${ }^{3}$ James Olson, ${ }^{5}$ and Constance L. Cepko ${ }^{1,2,6}$ \\ ${ }^{1}$ Department of Genetics, Harvard Medical School, Boston, Massachusetts 02115, ${ }^{2}$ Howard Hughes Medical Institute, ${ }^{3}$ Department of Neurogenetics, Max \\ Planck Institute of Experimental Medicine, D-37075 Goettingen, Germany, ${ }^{4}$ Institute of Cell Biology and Neurobiology, Charité-Universitätsmedizin Berlin, \\ Campus Mitte, D-10098 Berlin, Germany, ${ }^{5}$ Division of Clinical Research, Fred Hutchinson Cancer Research Center, Seattle, Washington 98109, and \\ ${ }^{6}$ Department of Ophthalmology, Harvard Medical School, Boston, Massachusetts 02114
}

Members of the basic helix-loop-helix (bHLH) family of transcription factors have been shown to control critical aspects of development in many tissues. To identify bHLH genes that might regulate specific aspects of retinal cell development, we investigated the expression of bHLH genes in single, developing mouse retinal cells, with particular emphasis on the NeuroD family. Two of these factors, NeuroD2 and NeuroD6/NEX, had not been previously reported as expressed in the retina. A series of loss- and gain-of-function experiments was performed, which suggested that NeuroD genes have both similarities and differences in their activities. Notably, misexpression of NeuroD genes can direct amacrine cell processes to two to three specific sublaminae in the inner plexiform layer. This effect is specific to cell type and NeuroD gene, as the AII amacrine cell type is refractory to the effects of NeuroD1 and NeuroD6, but uniquely sensitive to the effect of NeuroD2 on neurite targeting. Additionally, NeuroD2 is endogenously expressed in AII amacrine cells, among others, and loss of NeuroD2 function results in a partial loss of AII amacrine cells. The effects of misexpressing NeuroD genes on retinal cell fate determination also suggested shared and divergent functions. Remarkably, NeuroD2 misexpression induced ganglion cell production even after the normal developmental window of ganglion cell genesis. Together, these data suggest that members of the NeuroD family are important for neuronal cell type identity and may be involved in several cell type-specific aspects of retinal development, including fate determination, differentiation, morphological development, and circuit formation.

\section{Introduction}

The vertebrate retina is an excellent model for studying the development of cellular diversity in the CNS because it contains many functionally distinct and well characterized cell types (Masland, 2001). Retinal cells belong to one of five neuronal cell classes or to one glial cell class, and each class contains one or more distinct cell types. Although cells within a general class share broad characteristics, specific cell types have similar morphological, molecular, and physiological properties, which underlie their shared function (Masland, 2004). Developmentally, cells of the same type must attain these shared attributes through shared or convergent mechanisms. However, the retina must also generate the appropriate degree of cellular diversity to ensure that all of the distinct cell types are produced and project to their correct synaptic targets to form functional neural circuits. The

\footnotetext{
Received May 18, 2010; revised Feb. 14, 2011; accepted March 7, 2011.

Author contributions: T.J.C. and C.L.C. designed research; T.J.C., S.W., I.B., M.S., and J.0. performed research; T.J.C., S.W., and C.L.C. analyzed data; T.J.C. and C.L.C. wrote the paper.

This work was supported by NIH Grants EY008064 and EY009676. C.L.C. is an Investigator of the Howard Hughes Medical Institute, and S.W. is an Associate of the Howard Hughes Medical Institute. We thankmembers of the Cepko, Tabin, and Dymecki Laboratories, especially Dr. Jeffrey M. Trimarchi, for helpful discussions and advice. We are also grateful for discussions and advice from Drs. Elio Raviola and Richard Masland.

Correspondence should be addressed to Constance L. Cepko, Room 360, New Research Building, Department of Genetics, Harvard Medical School, 77 Avenue Louis Pasteur, Boston, Massachusetts 02115. E-mail: cepko@genetics.med.harvard.edu.

DOI:10.1523/JNEUROSCI.2555-10.2011

Copyright $\odot 2011$ the authors $\quad 0270-6474 / 11 / 317365-15 \$ 15.00 / 0$
}

molecular mechanisms that drive cell type-specific development, generate diversity across cell types, and form circuits to link these cell types are under investigation in several systems.

The basic helix-loop-helix (bHLH) transcription factors are one family of genes that has been shown to be important for the specification of distinct retinal cell classes ((Morrow et al., 1999; Inoue et al., 2002) and, in a few cases, for the development of specific cell types (Bramblett et al., 2004; Feng et al., 2006). The changing expression patterns of bHLH factors, along with other factors like homeodomain transcription factors, are thought to influence the competence of retinal progenitor cells to produce these cell types over the course of retinal development (Cepko, 1999; Hatakeyama and Kageyama, 2004). However, to generate the extensive diversity of $\sim 55$ retinal cell types, far more factors must be required than those that have been identified thus far.

The retina is also a powerful system for studying local neurite targeting because its cell types project in stereotyped patterns to well defined laminae within the retinal plexiform layers (Sanes and Zipursky, 2010). The bHLH family of transcription factors has been implicated in neurite outgrowth, target selection, and synaptogenesis elsewhere in the CNS (Gaudillière et al., 2004; Ince-Dunn et al., 2006; Yang et al., 2009). However, this function of bHLHs has not been explored in the retina. Several molecular mechanisms have recently been discovered that influence the patterning of retinal neurites and the lamination of the inner plexiform layer. These genes include adhesion molecules as well as repulsive transmembrane guidance cues and self-avoidance fac- 
tors (Yamagata et al., 2002; Fuerst et al., 2008, 2009; Matsuoka et al., 2011), but transcriptional regulators of these processes have yet to be identified.

The goal of this study was to systematically identify bHLHs that are differentially expressed during cell fate specification and among mature retinal cell types, and to explore their potential developmental roles. Expression analyses and functional studies of the NeuroD family of bHLH genes demonstrated that these factors can be functionally distinct from one another and have multiple roles, including aspects of cell fate specification and neurite targeting.

\section{Materials and Methods}

Analysis of single-cell profiles. Quality control of single-cell Affymetrix probe signals and data filters were established as previously described (Cherry et al., 2009). To identify bHLH transcription factors differentially expressed among distinct retinal cell types, probe sets were filtered against cell type identity according to unbiased clustering of cell types and known markers of individual cell types or classes (Trimarchi et al., 2008; Cherry et al., 2009).

Mice. Generation of neuroD1 and neuroD2 knock-out/lacZ knock-in mice and genotyping methods have been previously described (Miyata et al., 1999; Olson et al., 2001). Generation of the nex/neuroD6 knock-out/ cre knock-in mouse and the Rosa26 cre-sensitive lacZ reporter (R26R) mouse lines have been previously described (Soriano, 1999; Goebbels et al., 2006). CD1 mice were obtained from Charles River Laboratories. Both male and female mice were used in this study. All of the experiments in this study were approved by the Institutional Animal Care and Use Committee at Harvard University.

Histology. Mouse eyeballs were fixed for $2 \mathrm{~h}$ in $4 \%$ PFA in $1 \times \mathrm{PBS}, \mathrm{pH}$ 7.4. Whole retinae were then dissected free of the eyeball in $1 \times \mathrm{PBS}$. After dissection, retinae were equilibrated at room temperature in sucrose $/ 1 \times$ PBS solutions of increasing sucrose concentrations $(5,15,30 \%)$. Retinae were finally equilibrated for $>60 \mathrm{~min}$ in a well mixed 1:1 solution of OCT (Tissue-Tek; Sakura Finetek), and 30\% sucrose/PBS, and frozen quickly on dry ice. Retinal cryosections were cut $20 \mu \mathrm{m}$ thick using a disposable blade on a Leica CM3050S cryostat (Leica Microsystems).

Immunofluorescence and in situ hybridization. Retinal cryosections were blocked for $30 \mathrm{~min}$ in $0.1 \%$ Triton, $0.02 \%$ SDS, $1 \%$ BSA in $1 \times$ PBS. Sections were then incubated in a humidified chamber at room temperature (RT) overnight, with primary antibodies and DAPI (Sigma-Aldrich; D9542) diluted in blocking solution. Primary antibodies used in this study included the following: rabbit anti- $\beta$-galactosidase ( $\beta$ gal) (1: 250; Millipore Bioscience Research Reagents; AB1211), goat anti- $\beta$ galactosidase (1:800; Santa Cruz; sc-19119), chicken anti-GFP (1:2000; Abcam; AB13970), rabbit anti-Chx10 (1:500; C. L. Cepko Laboratory), mouse anti-p27 ${ }^{\text {Kipl }}$ (1:50; BD Biosciences Transduction Laboratories; clone 57), rabbit anti-Sox9 (1:750; Millipore; ab5535), rabbit anti-Pax6 (1:200; Covance; PRB-278P-100), goat anti-Brn3 (1:500; Santa Cruz; C-13), rabbit anti-Gad65/67 (1:250; Millipore Bioscience Research Reagents; AB5840), goat anti-GlyT1 (1:5000; Millipore Bioscience Research Reagents; AB1770), rabbit anti-Dab1 (1:250; Millipore Bioscience Research Reagents; AB5840), goat anti-ChAT (1:30; Millipore Bioscience Research Reagents; AB144P), rabbit anti-PKC $\alpha$ (1:1000; Sigma-Aldrich; P4334), rabbit anti-NeuroD2 (1:300; Abcam; ab31938), and mouse anticre recombinase (1:100; Covance; BIOT-106L). Slides were then washed three times in $1 \times$ PBS and incubated for $2 \mathrm{~h}$ with fluorescently coupled secondary antibodies (Jackson ImmunoResearch) and DAPI. Slides were washed three times with $1 \times$ PBS and mounted using Fluoromount-G (Southern Biotechnology Associates; 0100-01).

Whole retina immunostaining was achieved by microfracturing retinae with three rapid freeze-thaw cycles after retinae had been equilibrated in $30 \%$ sucrose $/ 1 \times$ PBS. The sucrose gradient steps were then reversed until the retina was equilibrated in $1 \times$ PBS. Retinae were blocked for $1 \mathrm{~h}$ in blocking solution, incubated with primary antibodies for 1-3 d at RT, washed three times in $1 \times$ PBS, incubated with secondary antibodies, washed again, and mounted in ProLong Gold (Invitrogen; P36930).
In situ hybridization on retinal sections and dissociated cells was performed as previously described (Trimarchi et al., 2007). For experiments with codetection of dissociated cell RNA in situ hybridization and 5-ethynyl-2-deoxyuridine (EdU) labeling, postnatal day 0 (P0) mouse pups were injected with $10 \mu \mathrm{g}$ of EdU per gram of animal. Retinae were harvested after $2 \mathrm{~h}$, dissociated, fixed to glass slides with $4 \%$ PFA in PBS, $\mathrm{pH} 7.4$, and stored at $-20^{\circ} \mathrm{C}$ in methanol until needed (Trimarchi et al., 2007). Detection of RNA by in situ hybridization was performed before detection of EdU. The tyramide reaction was developed for $15 \mathrm{~min}$. Slides were then washed three times in $1 \times$ PBS and postfixed with $4 \%$ PFA in PBS, pH 7.4. EdU labeling was detected with a Click-iT EdU Imaging Kit (Invitrogen; C10083). Slides were rinsed twice in 3\% BSA in PBS. Detection solution was applied directly to slides and slides were incubated in the dark for $30 \mathrm{~min}$ at room temperature. Slides were rinsed again in $3 \%$ BSA in PBS, incubated in DAPI in $1 \times$ PBS, rinsed in PBS, and coverslipped.

Microscopy and image analysis. Images were acquired using a Leica TCS SP5 confocal microscope. Images were analyzed, quantified, and uniformly adjusted for brightness, contrast, and smoothing using Imaris 5.7 software (Bitplane). Colocalization of molecular markers in individual cells was assessed by analysis of three-dimensional $Z$-series with Imaris 5.7 software.

Misexpression constructs and in vivo electroporation. The plasmid CAG: NeuroD1-ires-GFP was constructed by PCR amplification from a full-length mouse cDNA clone using NeuroD1, forward, 5' -ATATGAATTCATGACCAAATCATACAGCGAGA-3', and NeuroD1, reverse, 5' -ATATGCGGCCGCTAATCGTGAAAGATGGCATTAAG-3', primer sets and cloning into the CAGIG vector (Matsuda and Cepko, 2004), using EcoRI and NotI restriction sites. CAG:NeuroD2-ires-GFP was constructed using an identical strategy by PCR amplification of a full-length cDNA from EST BC058965 using NeuroD2, forward, $5^{\prime}$-ATATGAATTCATGCACCATGCTGACCCGCCTGTTC-3', and NeuroD2, reverse, 5'-ATATGCGGCCGCTCAGTTATGGAAAAATGCGTT-3', primer sets. CAG:NeuroD6-ires_GFP was cloned from a full-length mouse EST using NeuroD6, forward, 5' GTACTGAATTCTGACTACCGTTTGAC-3', and NeuroD6, reverse, 5'GATCGCGGCCGCCAATTATGAAAAAC-3' , primer sets. Each construct was verified by sequencing. CAGIG was electroporated alone for control experiments.

In vivo injection of expression constructs and electroporation was done on male and female pups exactly as previously described (Matsuda and Cepko, 2004) except for using an oocyte microinjector (Drummond; 3-000-510-X) or a Femtojet Express pressure injector (Eppendorf; 920010521) and pulled glass pipettes (Dumont/Drummond; 3000-210G8) or capillary pipette tips (Eppendorf; E5242956003) to deliver $\sim 0.1$ $\mu \mathrm{l}$ of $1 \mu \mathrm{g} / \mu \mathrm{l}$ DNA solution to the subretinal space of the developing mouse eye.

Statistical methods. To compare differences between control and experimental values for statistical significance, Student's two-tailed $t$ test was used for measurements based on categorical data (i.e., percentage of electroporated cells belonging to a given cell class).

\section{Results}

Developing retinal cell types express distinct atonal-like basic helix-loop-helix transcription factors

To determine whether individual bHLH transcription factors are differentially expressed among specific retinal cell types, microarray data from single profiled amacrine cells (Cherry et al., 2009) were analyzed. The dataset included single developing amacrine cells belonging to the major groups of the amacrine cell class, the GABAergic and glycinergic cells, as well as several specific amacrine cell types. GABAergic amacrine cells were identified according to their expression of Gad1. Glycinergic amacrine cells were identified according to their expression of GlyT1. Cholinergic amacrine cells were identified according to expression of VAChT. AII amacrine cells were identified according to expression of Dab1, Gjd2 (formerly known as Gja9), and Gtf2h4. Cholinergic and AII amacrine cell types were also identified independently 


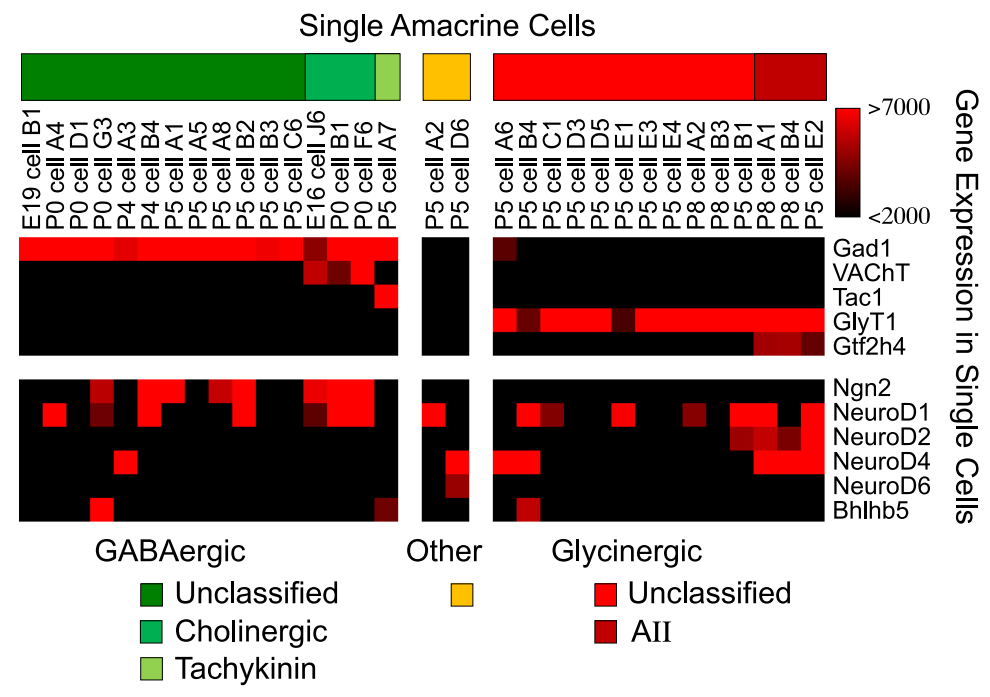

Figure 1. Distinct atonal-like basic helix-loop-helix transcription factors are expressed in specific amacrine cell types during development. A heat map generated using Treeview software of atonal-like bHLH expression in 32 individual amacrine cells profiled between $\mathrm{E} 16$ and P8. Single profiled amacrine cells belonging to GABAergic (green), glycinergic (red), and other (yellow) amacrine cell groups express distinct bHLH family members. Ngn2 is expressed in a subset of GABAergic amacrine cell types, but not glycinergic or other amacrine cell types. NeuroD1 is expressed in cholinergic and All amacrine cell types, among others. NeuroD2 expression is specific to All amacrine cells and one other glycinergic amacrine cell. NeuroD4/Math3 is expressed in All amacrine cells and is biased toward amacrine cells of the glycinergic group, but is expressed in other amacrine cells, including at least one type of GABAergic cell. NeuroD6 was expressed in one amacrine cell that did not express markers of GABAergic or glycinergic amacrine cells. Molecular classification of amacrine cell type was made based on expression of known amacrine cell type markers (Gad1 ${ }^{+}$ GABAergic; GlyT1 ${ }^{+}$, glycinergic; VAChT, cholinergic; Gtf2h4, All amacrine; Gad1 ${ }^{-} / \mathrm{GlyT1}^{-}$, other) and according to Pearson's correlation and Ward's clustering method of single-cell transcriptional profiles (Cherry et al., 2009).

through a previous unsupervised clustering study (Cherry et al., 2009). The dataset also included amacrine cells that did not express Gad1 or GlyT1. Different bHLH transcription factors belonging to the atonal-like superfamily were found to be expressed in distinct amacrine cell types.

The atonal-like superfamily of bHLH transcription factors is made up seven families: NeuroD, Neurogenin, Beta3, Oligo, Mist, Atonal, and Net (Ledent et al., 2002). Members of the NeuroD, Neurogenin, and Beta3 families were found to be expressed in distinct patterns among amacrine cell types (Fig. 1). Ngn2, a member of the neurogenin family, was expressed in $50 \%$ of GABAergic amacrine cells, but not in glycinergic or other amacrine cell types. Among the other GABAergic amacrine cells, Ngn2 was expressed in three of three cholinergic, starburst-type amacrine cells.

Members of the NeuroD family of atonal-like bHLH transcription factors are known to be important in development of the amacrine cell class (Morrow et al., 1999; Inoue et al., 2002), but little is known about their specific expression in individual cell types. We found distinct patterns of expression of different NeuroD factors among individual amacrine cell types (Fig. 1). NeuroD1 was expressed in cholinergic and AII amacrine cell types, among other GABAergic and glycinergic amacrine cells. In contrast, the expression of a closely related factor, NeuroD2, was exclusive to a small subset of glycinergic amacrine cells, including AII amacrine cells. NeuroD4/Math3 was expressed in 5 of 14 glycinergic amacrine cells, including AII amacrine cells, 1 of 2 GlyT1 ${ }^{-}$, Gad1 ${ }^{-}$amacrine cells, and 1 of 16 GABAergic cells. Although NeuroD1 was expressed in two of three AII amacrine cells, NeuroD2 and NeuroD4 were expressed in all three profiled AII amacrine cells. NeuroD6/Math2/Nex was expressed in one GlyT1 ${ }^{-}$, Gad1 ${ }^{-}$amacrine cell, but not in any GABAergic or glycinergic cells. Neither Ngn1/NeuroD3 nor Ngn3/Atoh5 were expressed in a serial analysis of gene expression study covering the development of the entire retina (Blackshaw et al., 2004 ) or in any of $>160$ profiles of single developing retinal cells (Trimarchi et al., 2007, 2008; Kim et al., 2008; Roesch et al., 2008; Cherry et al., 2009).

Members of the Beta3 family of bHLH transcription factors include Bhlhb4 and Bhlhb5. Bhlhb4 was not expressed in any amacrine cells, consistent with a previous report (Bramblett et al., 2004). Bhlhb5 has been reported to be expressed in amacrine and bipolar cells (Feng et al., 2006). In our dataset, Bhlhb5 was expressed by 2 of 16 GABAergic and 1 of 14 glycinergic amacrine cells (Fig. 1). Among the GABAergic cells, the only cell that expressed Tac1, a prepropeptide gene for substance $\mathrm{P}$ and neurokinin A, also expressed Bhlhb5.

Expression of Ngn2, NeuroD1, NeuroD4/Math3, and Bhlhb5 in the retina has been previously reported (Lee et al., 1995; Gradwohl et al., 1996; Sommer et al., 1996; Morrow et al., 1999; Inoue et al., 2002; Feng et al., 2006), whereas expression of NeuroD2 and NeuroD6 in the retina has not been demonstrated. To verify the expression of these bHLH factors, RNA in situ hybridization of retinal tissue at developmental and mature time points was performed. NeuroD6 was expressed in the developing inner neuroblastic layer at embryonic day 18 (E18), whereas NeuroD4/Math3 was expressed in the outer neuroblastic layer (supplemental Fig. 1, available at www.jneurosci.org as supplemental material). By P6, NeuroD2 was expressed in a pattern consistent with expression in amacrine cells, in the lower portion of the inner nuclear layer (INL). In the mature retina, NeuroD2 was expressed in a subset of amacrine cells in the lower portion of the INL and a subset of cells in the ganglion cell layer (GCL). NeuroD6 continued to be expressed in the developing GCL at P6 but also expanded its expression to the INL and horizontal cells. In the adult retina, NeuroD6 appeared to be expressed in the GCL and INL. Strong NeuroD6 signal was apparent at the apical border of the retina as well.

To further investigate the differential expression of bHLH family transcription factors in specific types of cells in the retina, we focused on NeuroD1, NeuroD2, and NeuroD6, three closely related bHLH family members.

\section{NeuroD factors define distinct retinal cell types within the major cell classes of the retina}

To verify the expression of NeuroD genes in specific retinal cell types, we made use of three previously generated mouse lines, a NeuroD1 knock-out/lacZ knock-in line (Miyata et al., 1999; Morrow et al., 1999), a NeuroD2 knock-out/lacZ knock-in line (Olson et al., 2001), and a NeuroD6/NEX knock-out/cre recombinase knock-in line (Schwab et al., 2000) bred to the Rosa26 cre-sensitive lacZ reporter (R26R) (Soriano, 1999). Although these reporter lines have been demonstrated to recapitulate endogenous gene expression, they only reflect transcriptional activity at these genetic loci. The respective reporters are not representative of posttranscriptional regulation. Despite this, we chose to analyze the knock-in mice as proxies for NeuroD factor expres- 


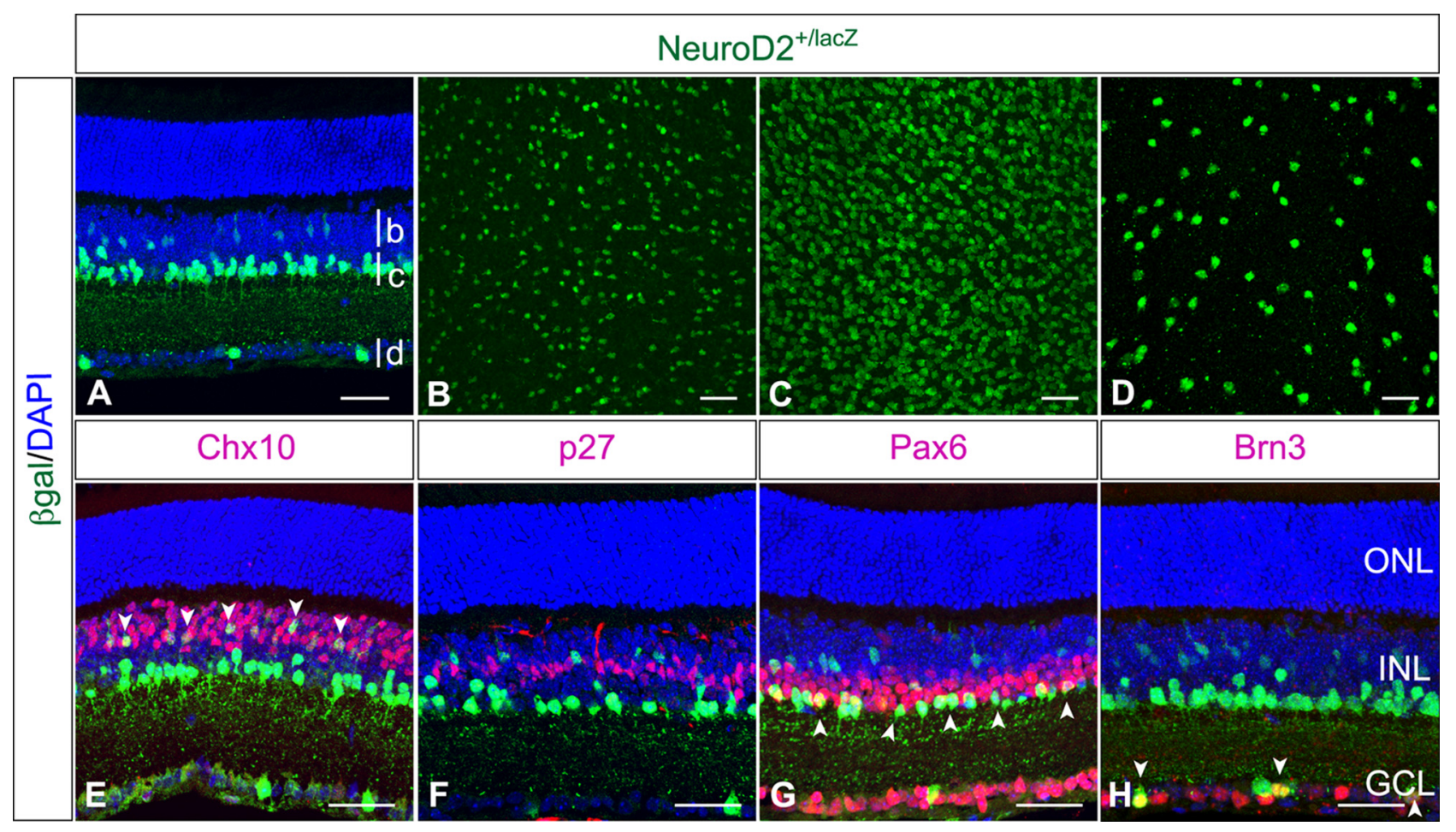

Figure 2. NeuroD2 is expressed in amacrine, ganglion, and bipolar cells of the mature mouse retina. The expression of NeuroD2 was examined by immunofluorescent detection of $\beta$ gal in mice heterozygous for the NeuroD2 knock-out/lacZ knock-in allele. A, At P22, NeuroD2 is expressed in cells of the upper (b) and lower (c) INL and GCL (d) of the retina, as seen in cross section. $\boldsymbol{B}$ - $\boldsymbol{D}$ correspond to three horizontal planes of the retina at the level of b-d, respectively. $\boldsymbol{B}, \beta \mathrm{gal}{ }^{+}$cells in the horizontal plane of the upper INL. $\boldsymbol{C}, \beta \mathrm{gal}{ }^{+}$cells in the horizontal plane of the lower INL. $\mathbf{D}, \beta \mathrm{gal}{ }^{+}$cells in the horizontal plane of the GCL. $\boldsymbol{E}$, NeuroD2 (green) is expressed in a subset of $\mathrm{Chx} 10^{+}$(red) bipolar cells. $\boldsymbol{F}$, NeuroD2 (green) is not expressed in p27 ${ }^{+}$(red) Müller glial cells. $\mathbf{G}$, NeuroD2 is expressed in a subset of $\mathrm{Pax}^{+}{ }^{+}$(red) amacrine cells in the lower INL. $\boldsymbol{F}$, NeuroD2 is expressed in a subset of Brn ${ }^{+}$(red) ganglion cells in the GCL. DAPI, Blue. In $\boldsymbol{E}, \boldsymbol{G}$, and $\boldsymbol{H}$, the arrowheads point to double-positive cells. Scale bars, $40 \mu \mathrm{m}$.

sion because reporter expression revealed the morphology of individual cells, and because currently available NeuroD2 antibodies showed a marked lack of specificity to their proposed epitopes (supplemental Fig. 2, available at www.jneurosci.org as supplemental material).

Differential expression of bHLH transcription factors in distinct types of amacrine cells may occur transiently during development or as part of an enduring cell type-specific pattern. To distinguish between these possibilities in the case of NeuroD1 and NeuroD2, we analyzed heterozygous retinae containing one lacZ knock-in allele from developmental (P6) and mature (P22) time points. At P6, NeuroD1 was expressed in photoreceptors of the outer nuclear layer (ONL), in a subset of cells in the lower half of the INL, and in a subset of cells in the GCL. The pattern of expression in the INL and GCL was suggestive of amacrine cells and displaced amacrine cells or ganglion cells (supplemental Fig. $3 A$, available at www.jneurosci.org as supplemental material). By P22, NeuroD1 expression had expanded to include cells in the upper half of the INL, whose processes projected to the ON strata of the inner plexiform layer (IPL) corresponding to ON bipolar cells (supplemental Fig. 3B, available at www.jneurosci.org as supplemental material). Expression in photoreceptors and a subset of lower INL and GCL cells continued. NeuroD2 was expressed in a subset of cells in the lower INL and in a subset of cells in the GCL at P6 (supplemental Fig. 3C, available at www. jneurosci.org as supplemental material). NeuroD2 expression at P22 was similar to its expression at P6, but included cell bodies in the central portion of the INL (supplemental Figs. $2 A, B, 3 D$, available at www.jneurosci.org as supplemental material). NeuroD2-expressing cells in the central portion of the INL colo- calized with Chx10, a marker for the bipolar cell class, but not with p27, a marker for Müller glial cells (Fig. 2 E, F; supplemental Fig. $4 A-H$, available at www.jneurosci.org as supplemental material). In the lower INL, NeuroD2-expressing cells were a subset of Pax $6^{+}$cells belonging to the amacrine cell class (Fig. 2G; supplemental Fig. $4 I-L$, available at www.jneurosci.org as supplemental material). In the GCL, a subset of NeuroD2-expressing cells expressed Brn3, a marker for the ganglion cell class (Fig. $2 \mathrm{H}$; supplemental Fig. $4 M-P$, available at www.jneurosci.org as supplemental material).

In the case of NeuroD6, it was possible to distinguish between transient and mature persistent expression by taking advantage of the cre-sensitive lac $Z$ reporter and heterozygous mice for the NeuroD6:cre allele. Cells that have a history of NeuroD6:cre expression and their daughters will be labeled by cre-mediated recombination of the lac $\mathrm{Z}$ reporter allele and therefore express $\beta$ gal. Cells that actively express NeuroD6 will also be immunoreactive for cre, which is driven directly by the NeuroD6 promoter. In contrast, cells that have a history of NeuroD6 expression, but no longer actively express NeuroD6, will be $\beta \mathrm{gal}^{+}$, but $\mathrm{cre}^{-}$.

In the mature retina, NeuroD6 fate mapped cells included cells in the upper part of the INL, just below the outer plexiform layer (OPL), cells in the inner portion of the INL, and cells in the GCL (Fig. 3A-H). Cells actively expressing cre from the NeuroD6 locus were found in the inner half of the inner nuclear layer (Fig. $3 D)$. According to known molecular markers, neither Chx10expressing bipolar cells nor p27-expressing Müller glia have a history of NeuroD6 expression (Fig. 3E,F; supplemental Fig. $5 A-H$, available at www.jneurosci.org as supplemental material). NeuroD6 does, however, fate map Pax $6^{+}$horizontal cells in the 


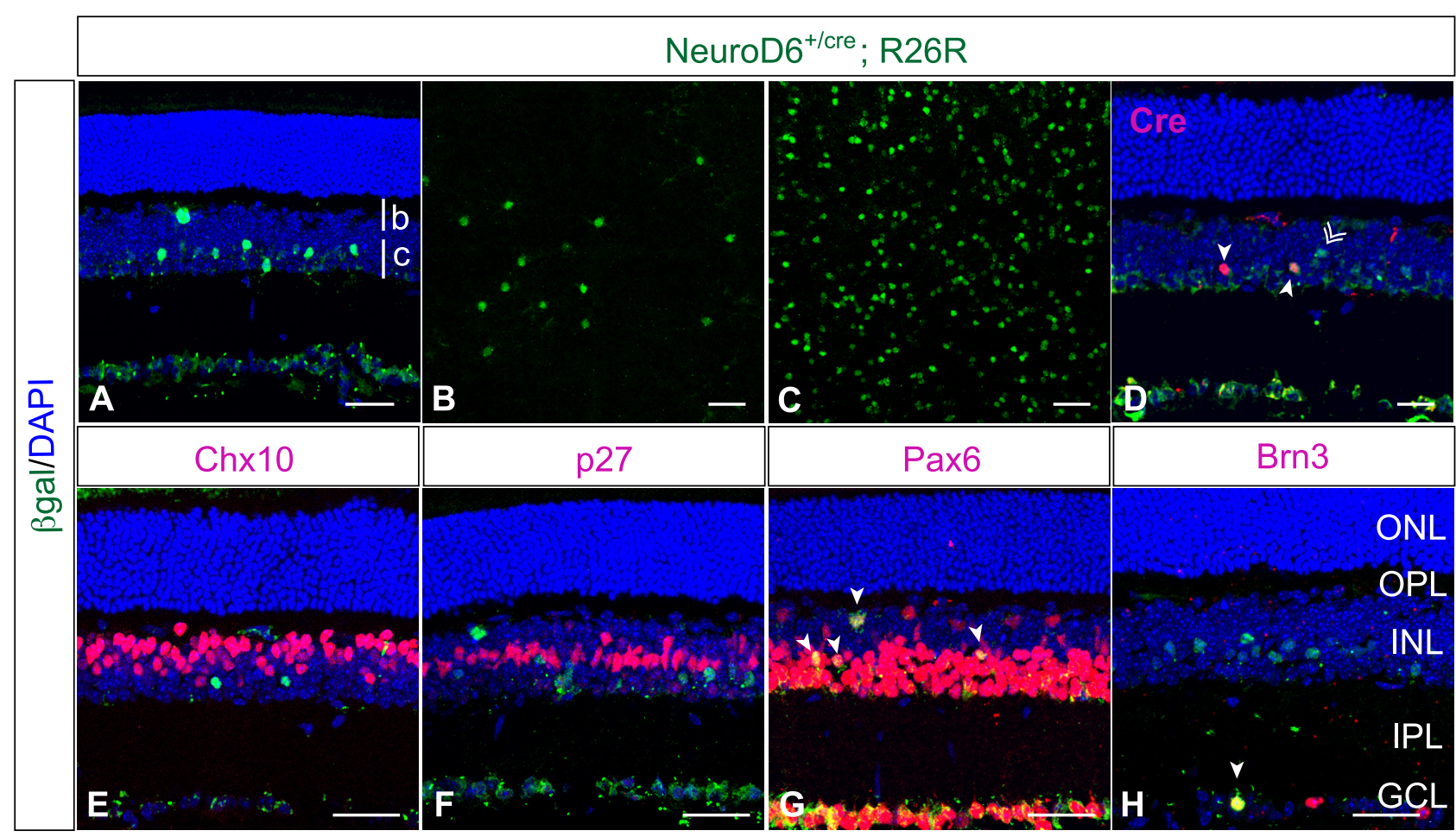

Figure 3. NeuroD6:cre fate maps horizontal, amacrine, and ganglion cells of the mature mouse retina. The fate of NeuroD6-expressing cells was examined by immunofluorescent detection of $\beta$ gal in mice heterozygous for the NeuroD6 knock-out/cre knock-in allele and the Rosa26 lacZ reporter of cre activity (R26R). A, At P22, NeuroD6 fate-mapped cells are found in the upper (b) and lower (c) INL, as seen in cross section. $\boldsymbol{B}$ and $\boldsymbol{C}$ correspond to two horizontal planes of the retina at the level of b and c, respectively. $\boldsymbol{B}, \beta \mathrm{gal}{ }^{+}$cells in the horizontal plane of the upper INL directly below the OPL. C, $\beta \mathrm{gal}^{+}{ }^{+}$cells in the horizontal plane of the lower INL directly above the IPL. D, A subset of fate-mapped cells in the lower INL continue to express (re-recombinase from the NeuroD6 allele at P22, as seen using immunofluorescence for Cre (red). $\boldsymbol{E}$, NeuroD6 fate-mapped cells expressing $\beta$ gal (green) do not correspond to Chx $10^{+}$(red) bipolar cells. $\boldsymbol{F}$, NeuroD6 does not fate map (green) $\mathrm{p} 27^{+}$(red) Müller glial cells. G, NeuroD6 does fate map a subset of Pax6 ${ }^{+}$(red) horizontal cells in the upper INL and a subset of Pax6 ${ }^{+}$amacrine cells in the lower INL. $\boldsymbol{H}$, NeuroD6 fate maps a small subset of Brn3 ${ }^{+}$(red) ganglion cells in the GCL. DAPI, Blue. In $\mathbf{D}, \mathbf{G}$, and $\boldsymbol{H}$, the arrowheads point to double-positive cells. In $\boldsymbol{D}$, the chevron points to a $\beta$ gal ${ }^{+}$(green) cell that no longer expresses (re (red). Scale bars, $40 \mu \mathrm{m}$.

upper INL, Pax6 ${ }^{+}$amacrine cells in the inner INL, and Brn3 ${ }^{+}$ ganglion cells in the GCL (Fig. 3G,H; supplemental Fig. 5I-P, available at www.jneurosci.org as supplemental material). Expression of NeuroD6 in the mature retina persists mainly in amacrine cells of the INL (Fig. 3D; supplemental Fig. 5Q-T, available at www.jneurosci.org as supplemental material).

Although NeuroD1, NeuroD2, and NeuroD6 were expressed in many of the major cell classes of the retina, they were not expressed in every cell type within each of these major classes. To determine whether functionally distinct cell types express one or more NeuroD factors, we colocalized $\beta$ gal, expressed from either the NeuroD1 or the NeuroD2 locus, or Cre expression from the NeuroD6 locus, with markers of molecularly defined cell types. NeuroD1-expressing cells in the upper INL appeared to be ONbipolar cells according to the location of their axon termini in the ON layers of the INL (supplemental Fig. 3B, available at www. jneurosci.org as supplemental material). Among ON-bipolar cells, PKC $\alpha$ is expressed by the rod bipolar cell type. Many of the NeuroD $1{ }^{+}$bipolar cells expressed PKC $\alpha$ (Fig. $4 A$; supplemental Fig. 6A-D, available at www.jneurosci.org as supplemental material). Within the lower half of the INL, a subset of NeuroD1 ${ }^{+}$ cells expressed Dab1, confirming our single-cell profiling results, which showed that NeuroD1 is expressed in the AII amacrine cell type (Fig. $4 B$; supplemental Fig. $6 E-H$, available at www. jneurosci.org as supplemental material). However, the strongest NeuroD1-expressing amacrine cells were cholinergic starburst amacrine cells, according to their colocalization with ChAT (Fig. $4 C$; supplemental Fig. 6I-L, available at www.jneurosci.org as supplemental material).
NeuroD2-expressing cells in the central INL expressed Chx10 (Fig. $2 E$ ), but not PKC $\alpha$ (Fig. $4 E$; supplemental Fig. 7A-D, available at www.jneurosci.org as supplemental material), demonstrating that they are cone bipolar cells. In the inner half of the INL, NeuroD2 was expressed in all Dab1 ${ }^{+}$AII amacrine cells and in at least one additional amacrine cell type (Fig. 4 F; supplemental Fig. $7 E-H$, available at www.jneurosci.org as supplemental material). Although little is known about this additional cell type, these cells were one of the amacrine cell types that express PKC $\alpha$ (Fig. $4 E$; supplemental Fig. $7 A-D$, available at www.jneurosci.org as supplemental material). In contrast to NeuroD1, NeuroD2 was not expressed in cholinergic amacrine cells (Fig. 4G; supplemental Fig. 7I-L, available at www.jneurosci.org as supplemental material). Expression of NeuroD2 in the GCL was in a subset of $\mathrm{Brn}^{+}$ganglion cells, as well as some cells that were not immunoreactive to a Brn3 antibody (Fig. $2 \mathrm{H}$; supplemental Fig. $4 \mathrm{M}-\mathrm{P}$, available at www.jneurosci.org as supplemental material).

NeuroD6 fate-mapped cells did not correspond to any single identified type of amacrine cell. A subset of these cells were GlyT1 ${ }^{+}$glycinergic amacrine cells (Fig. 4I; supplemental Fig. $8 A-D$, available at www.jneurosci.org as supplemental material). However, among Gad65/67 ${ }^{+}$amacrine cells, none was visibly fate-mapped according to the R26R- $\beta$ gal reporter (Fig. $4 \mathrm{~J}$; supplemental Fig. $8 E-H$, available at www.jneurosci.org as supplemental material). Cells in which NeuroD6 expression persisted in the mature retina corresponded to amacrine cells that did not appear to express either GlyT1 or Gad65/67 (Fig. 4 K; supplemental Fig. 8I-N, available at www.jneurosci.org as supplemental material), in keeping with the microarray data. 


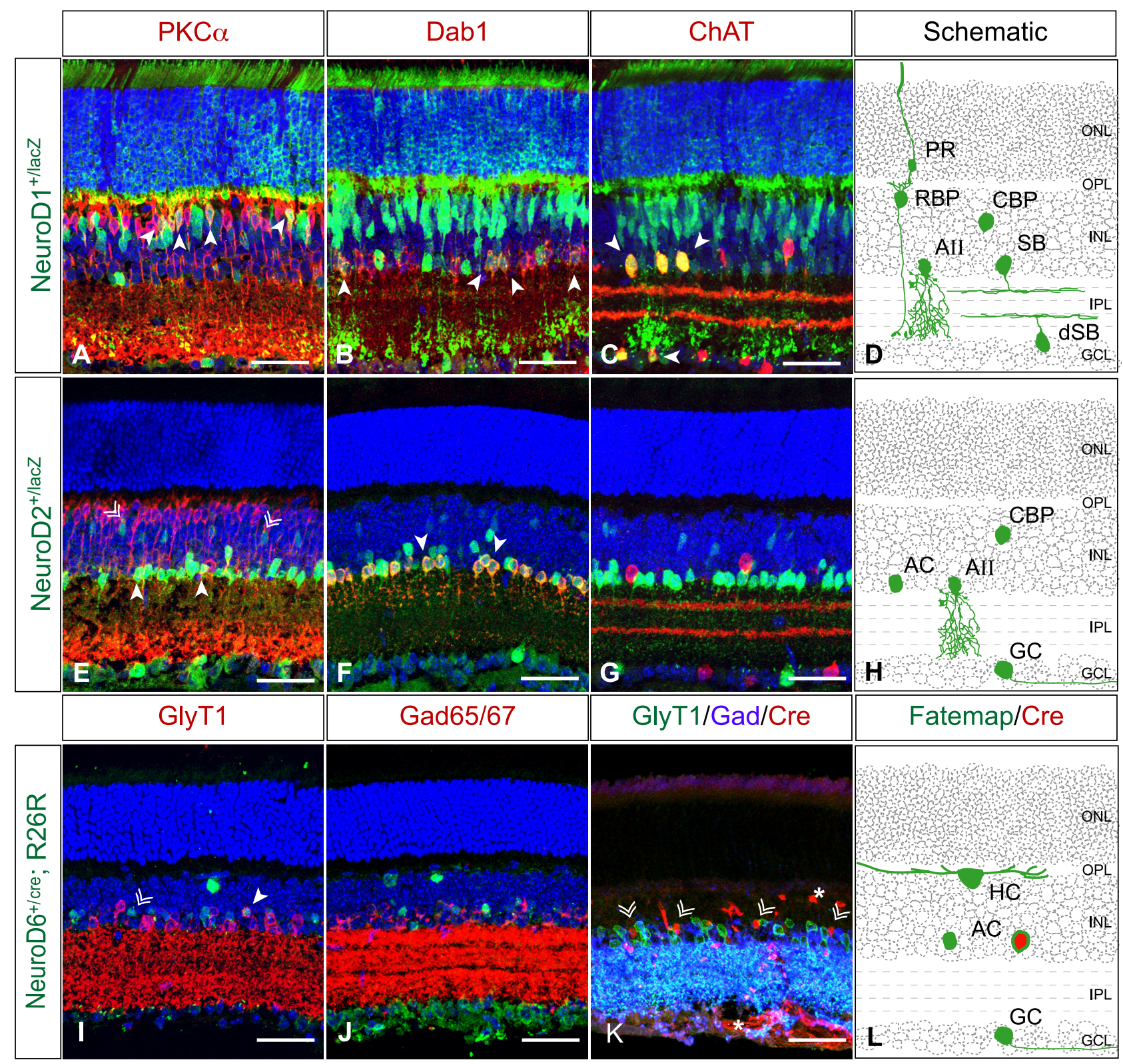

Figure 4. NeuroD1, NeuroD2, and NeuroD6 expression is specific to distinct neuron cell types of the mature retina. The expression of NeuroD1 $(\boldsymbol{A}-\boldsymbol{D})$ or NeuroD2 $(\boldsymbol{E}-\boldsymbol{H})$ was examined using immunofluorescent detection of $\beta$ gal in mice heterozygous for the NeuroD1 or NeuroD2 knock-out/lacZ knock-in allele. The fate map of NeuroD6-expressing cells or constitutive expression of NeuroD6 was examined using immunofluorescent detection of $\beta$ gal in mice heterozygous for the NeuroD6:cre allele and hemizygous for the R26R lacZ cre-responsive allele $(I, J)$ or by immunofluorescent detection of cre, respectively $(\boldsymbol{K})$. Specific cell types were defined according to immunoreactivity to cell type-specific markers. $\boldsymbol{A}$, At P22, NeuroD1 (green) is expressed in PKC $\alpha^{+}$(red) rod bipolar cells (RBPs) (white arrowheads). $\boldsymbol{B}$, NeuroD1 is expressed in Dab1 ${ }^{+}$(red) All amacrine cells (white arrowheads). C, NeuroD1 is also expressed in ChAT ${ }^{+}$(red) starburst (SB) amacrine cells in the INL (white arrowheads). NeuroD1-expressing cells (green) in the $\mathrm{GCL}$ are $\mathrm{ChAT}^{+}$(red) displaced starburst amacrine cells (dSB) (white arrowheads). $\boldsymbol{D}, A$ schematic of retinal cell types expressing NeuroD1. Three of these cells, rod photoreceptors (PR), RBPs, and All amacrine cells comprise the first part of the rod circuit. NeuroD1 is also expressed in at least one unidentified type of PKC $\alpha^{-}$cone bipolar cell (CBP) and SB and dSB amacrine cells. E, NeuroD2 is not expressed in PKC $\alpha^{+}$(red) RBP cells (white chevrons), although in the lower INL it is expressed in a subset of PKC $\alpha{ }^{+}$ (red) amacrine cells (white arrowheads). $\boldsymbol{F}$, Dab1 ${ }^{+}$All amacrine cells (red) express NeuroD2 (green) (white arrowheads). $\mathbf{G}, \mathrm{ChAT}^{+}$amacrine cell types do not express NeuroD2. $\boldsymbol{H}, \mathbf{A}$ schematic of retinal cell types that express NeuroD2. NeuroD2 is expressed in at least one type of PKC $\alpha^{-}$cone bipolar cell, All amacrine cells, at least one type of PKC $\alpha^{+}$, non-All amacrine cell, and at least one ganglion cell type (GC) (see Fig. 2 Hfor GC staining). I, A subset of NeuroD6:cre fate-mapped cells (green) express GlyT1 (red) (white arrowhead). Another subset of NeuroD6 fate-mapped cells (green) in the inner INL do not (white chevron). J, NeuroD6:cre fate-mapped cells (green) do not express Gad65/67 (red). $\boldsymbol{K}$, Cells actively expressing cre from the NeuroD6 locus (red) (white chevrons) [the asterisk $\left(^{*}\right.$ ) denotes background in vasculature] do not express GlyT1 (green) or Gad65/67 (blue). $L$, A schematic of retinal cells fate mapped by NeuroD6:cre (green) and cells actively expressing NeuroD6:cre in the mature retina. Horizontal cells, at least one type of glycinergic amacrine cell, at least one type Gly $\mathrm{T}^{-}{ }^{-} / \mathrm{Gad}^{-}$amacrine cell, and at least one type of ganglion cell are fate mapped by NeuroD6:cre (green and red) (see Fig. $3 \mathrm{H}$ for GC staining). At least one type GlyT1 ${ }^{-} / \mathrm{Gad}^{-}$amacrine cell (red) is actively expressing NeuroD6 in the adult retinal. DAPI, Blue. Scale bars, $40 \mu \mathrm{m}$.

\section{Cell type-specific bHLHs are expressed in retinal progenitor cells}

Members of the atonal-like superfamily of bHLHs that were seen in specific populations of postmitotic retinal neurons also were observed in mitotic retinal progenitor cells throughout develop- ment (Fig. 5A). These cells were profiled and identified as progenitor cells in a previous study (Trimarchi et al., 2008). Among progenitor cells, members of the Neurogenin, NeuroD, and Beta3 bHLH families were observed. Ngn2 and NeuroD1 were previously reported to be widely expressed among these progen- 
A

Retinal Progenitor Cells

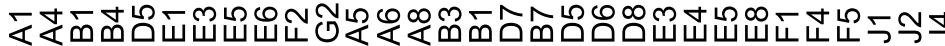

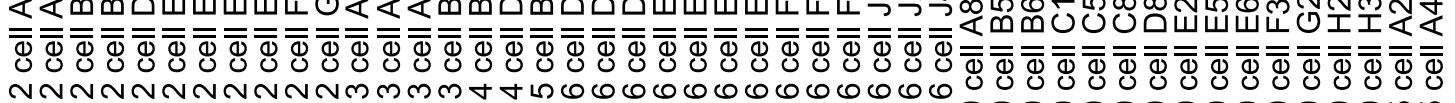

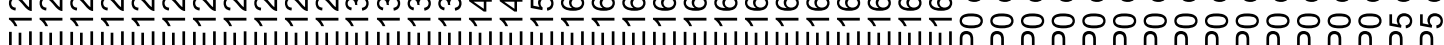
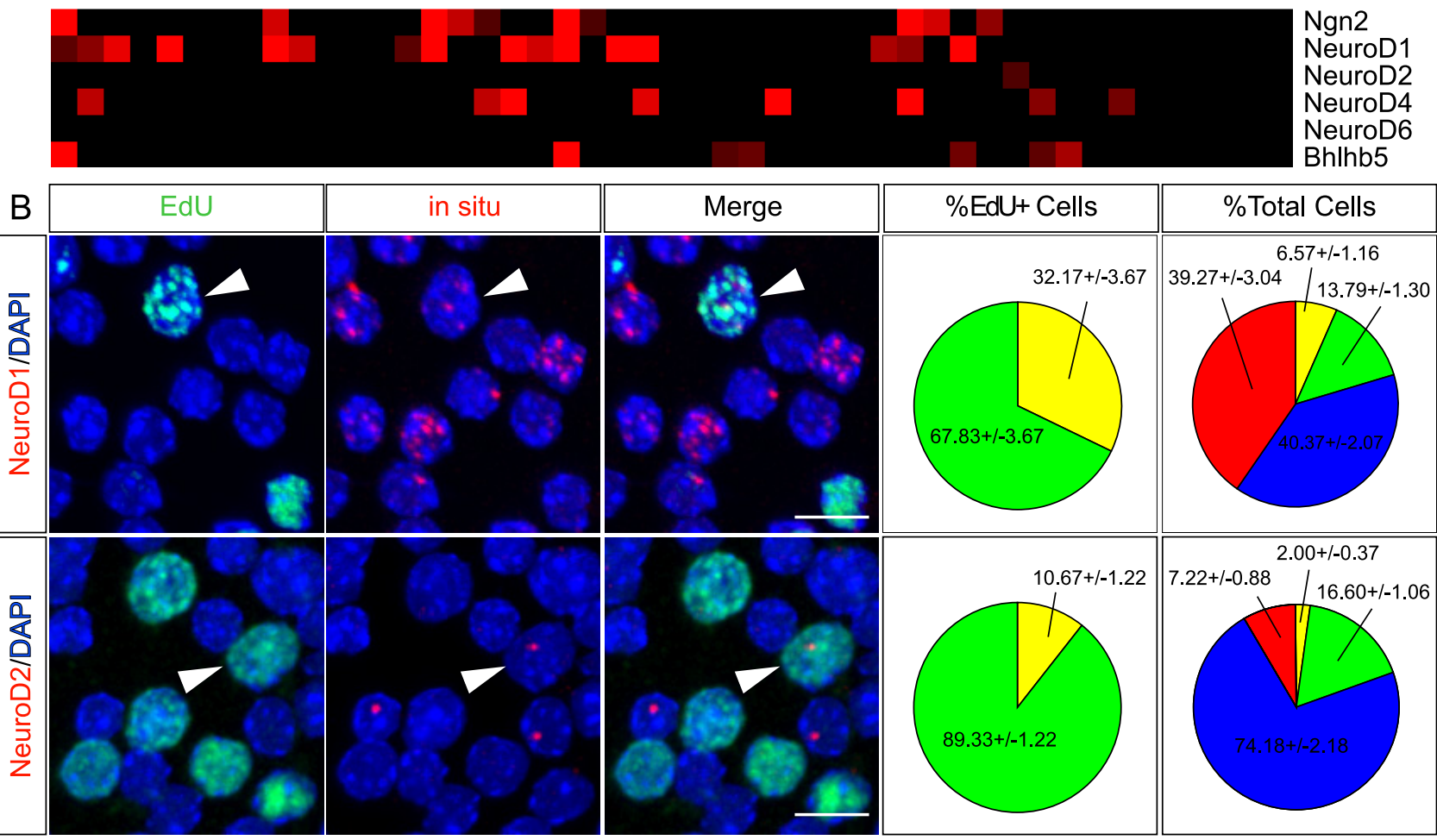

Figure 5. Cell type-specific bHLH expression in retinal progenitor cells. A, A heat map of bHLH transcription factor expression in single profiled retinal progenitor cells. Atonal-like bHLH factors that were expressed in specific subsets of developing amacrine cells are expressed in individually profiled retinal progenitor cells between E12 and P5. Ngn2, NeuroD1, NeuroD4/Math3, and Bhlhb5 were widely expressed in retinal progenitor cells throughout development. NeuroD2 was only expressed in one progenitor cell, at P0. NeuroD6 was not expressed in these cells. These cells were previously identified as progenitor cells according to known marker expression and a post hoc classification based on clusters of coexpressed genes centered around known markers of retinal progenitor cells (Trimarchi et al., 2008). B. Retinae were labeled in vivo at P0 with a pulse of EdU (green) and a $2 \mathrm{~h}$ chase, and then subjected to dissociation and RNA in situ hybridization for NeuroD1 or NeuroD2 (red). NeuroD1 and NeuroD2 were expressed in a subset of EdU ${ }^{+} \mathrm{P} 0$ retinal progenitor cells in, or recently in, S-phase of the cell cycle; however, most EdU ${ }^{+}$cells did not express NeuroD1 or NeuroD2. There were also many NeuroD1- or NeuroD2-expressing cells that were not labeled by EdU. These cells are either postmitotic cells or progenitor cells that were not in S-phase during the time of EdU labeling. The green and yellow pie charts show the percentage of EdU-labeled cells that express a NeuroD factor (yellow) or do not express NeuroD factors (green). The multicolored pie charts show the percentage of total cells that were labeled with EdU and express NeuroD factors (yellow), that were labeled with EdU, but do not express NeuroD factors (green), that express NeuroD factors, but were not EdU ${ }^{+}$(red), or cells that were not labeled by EdU or NeuroD ${ }^{+}$(blue). NeuroD1: $n=3$ retinae, $>380$ cells were counted per retina. NeuroD2: $n=3$ retinae, $>1370$ cells were counted per condition. Scale bar, $10 \mu \mathrm{m}$.

itor cells (Trimarchi et al., 2008). NeuroD2 was expressed in one progenitor cell, and NeuroD4 and Bhlhb5 were expressed in many progenitor cells. Of the atonal-like bHLHs that were differentially expressed in postmitotic amacrine cells, only NeuroD6, which was expressed in 1 of 32 amacrine cells, was not observed in the progenitor cell profiles.

To confirm the microarray data regarding expression of these factors in progenitor cells, retinae were labeled with EdU, a thymidine analog that is incorporated into progenitor cells during S-phase (Salic and Mitchison, 2008), and RNA in situ hybridization was performed. At P0, NeuroD1 was expressed in approximately one-third of retinal progenitor cells labeled in vivo with EdU (Fig. $5 B$ ). NeuroD2 was expressed in $\sim 11 \%$ of EdU-labeled cells. At P0, NeuroD1 - or NeuroD2-expressing cells that were not labeled by EdU were likely postmitotic cells or mitotic cells that were not in S-phase during the $2 \mathrm{~h}$ labeling period (Fig. $5 B$ ).

Expression of bHLHs in retinal progenitor cells suggests that they may be active in these cells, or poised to act in the newly postmitotic daughter cells in cell type specification or differenti- ation programs. The function of NeuroD1 in retinal development has been well characterized (Morrow et al., 1999; Inoue et al., 2002), so we focused additional functional investigations into bHLH function on NeuroD2 and NeuroD6, whose role has not been previously investigated in retinal cell type specification, differentiation, or maintenance.

\section{Loss of NeuroD2 results in a cell type-specific decrease in} cell number

To investigate the role of NeuroD2 during retinal development, we analyzed NeuroD $2^{\text {lacZ/lacZ }}$ mice and compared them with wild-type and heterozygous littermates. To visualize cells that would normally express NeuroD2, we made use of $\beta$-galactosidase expression in the heterozygous and homozygous null mice. Comparing heterozygous NeuroD2 $2^{+/ l a c z}$ retinae to NeuroD2 ${ }^{\text {lacZ/lacZ }}$ retinae, we observed a slight decrease in $\beta \mathrm{gal}^{+}$ cells in NeuroD2 $2^{\text {lacZ/lacZ }}$; however, the difference in cell number could not be distinguished from variation attributable to chance (Fig. 6A-C). 


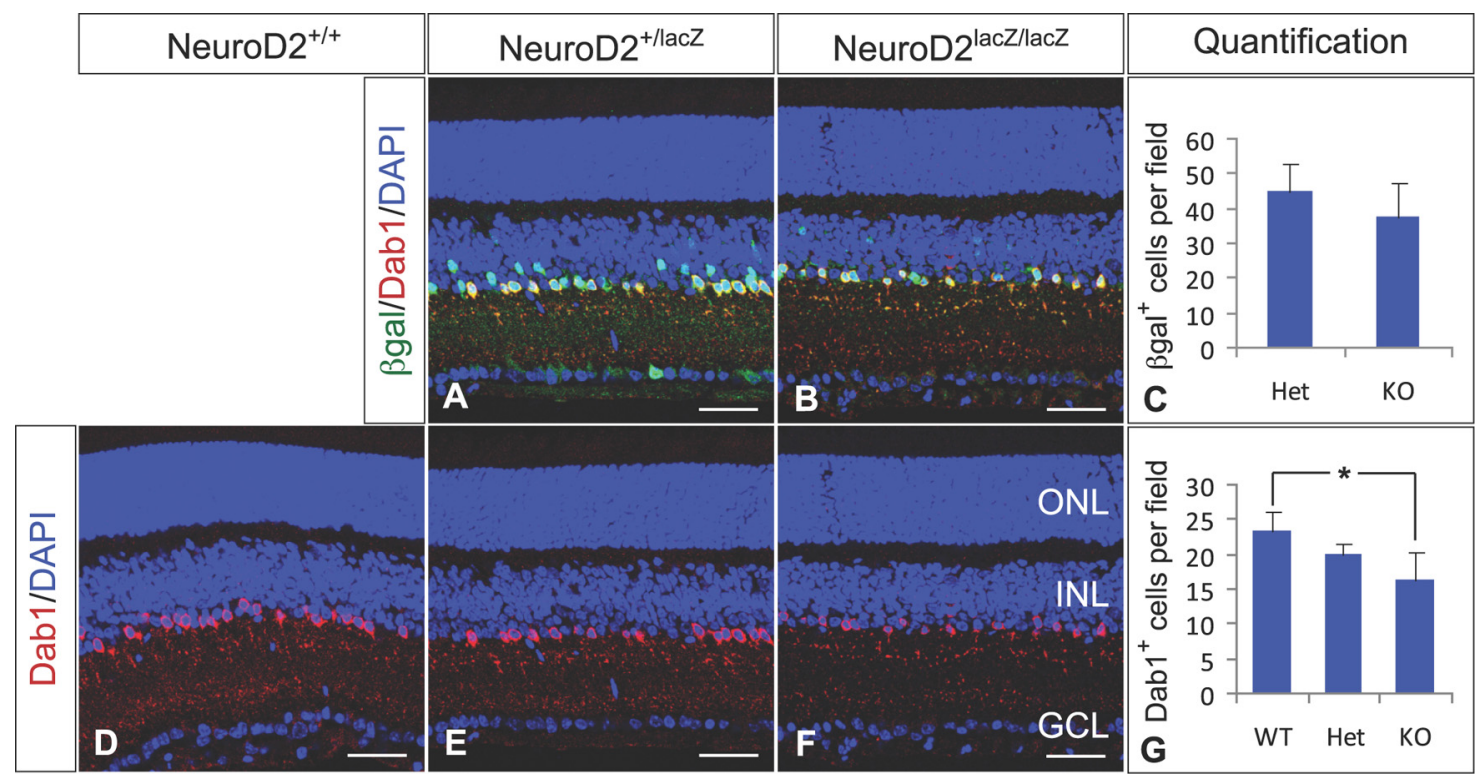

Figure 6. NeuroD2 inactivation results in a reduction in All amacrine cells. The effects of losing one or both alleles of NeuroD2 on retinal cells were analyzed by immunofluorescent detection of $\beta$ gal from NeuroD2 knock-out/lacZ knock-in alleles, or by cell type-specific markers in wild type (wt), heterozygous (het), and null littermates. $A, \beta$ gal-expressing cells (green), including Dab1 ${ }^{+}$ All amacrine cells (red), in a NeuroD2 heterozygous retina. $\boldsymbol{B}, \beta$ gal-expressing cells (green) including Dab1 ${ }^{+}$All amacrines (red) in a NeuroD2 null retina. $\boldsymbol{C}$, The number of $\beta$ gal-expressing cells per $375 \times 375 \mu \mathrm{m}$ field was quantified in NeuroD2 heterozygous and null retinae and compared using Student's $t$ test, two-tailed, homoscedastic, $p=0.27 . \mathbf{D}-\boldsymbol{F}$, Dab1 ${ }^{+}$All amacrine cells (red) in wt, het, and NeuroD2 null retinae. G, Numbers of All amacrine cells were compared between wt, het, and NeuroD2 null retinae, ${ }^{*} p=0.008 . C, G, n \geq 3$ retinae for each genotype and $\geq 2$ fields per retina. DAPI, Blue. Scale bar, $40 \mu \mathrm{m}$. Error bars indicate SD.

To determine what effect the loss of NeuroD2 may have on specific cell types that normally express NeuroD2, we investigated $\mathrm{Dab1}^{+}$AII amacrine cells in wild-type, heterozygous, and homozygous NeuroD2-null retinae. In comparing retinae from wild type, NeuroD2 $2^{+/ l a c Z}$, and NeuroD2 $2^{\text {lacZ/lacz }}$ littermates, we observed a decrease in the number of AII amacrine cells (Fig. $6 D-G)$, but no decrease in the numbers of $\mathrm{ChAT}^{+}$amacrine cells, which do not express NeuroD2 (data not shown). The difference in number of Dab1 ${ }^{+}$AII amacrine cells between wildtype and NeuroD2 $2^{\text {lacZ/lacz }}$ retinae was statistically significant $(p=0.008)$.

In the cerebellum, NeuroD2 is necessary for granule cell survival (Olson et al., 2001). To determine whether the cell loss in NeuroD2 null retinae was the result of cell death, we performed immunofluorescence for anti-cleaved caspase 3. At P22, very few anti-cleaved caspase 3 -immunoreactive cells were observed in any retina. It was possible, however, that the period of cell death preceded this time point, and so we also analyzed wild-type, NeuroD2 heterozygous, and knock-out retinae at an earlier postnatal time point (P6). The decrease in numbers of Dab1 ${ }^{+}$and $\beta g a l^{+}$ positive cells in NeuroD2 $2^{\text {lacZ/lacZ }}$ retinae, compared with wildtype and heterozygous retinae was already observable by this earlier time point (supplemental Fig. 2, available at www.jneurosci. org as supplemental material) (data not shown).

\section{Forced expression of individual NeuroD family members at P0 had differential effects on neuronal cell fate and suppressed gliogenesis}

The distinct expression patterns of each NeuroD family member may impart a unique role for each gene in the developing and mature retina. Additionally, the individual properties of the NeuroD proteins themselves may influence cell specification or differentiation in distinct ways. To test this latter possibility, each NeuroD family member was misexpressed individually under a ubiquitous promoter in the developing retina by in vivo electro- poration. This allowed for a direct comparison of NeuroD family member function in equivalent populations of cells. A bicistronic construct expressing NeuroD1, NeuroD2, or NeuroD6 and GFP (i.e., NeuroD1-ires-GFP) or a control plasmid expressing GFP alone (CAGIG) was electroporated into the retina at P0. Injection of plasmid solution into the subretinal space followed by electroporation drove DNA into retinal progenitor cells (Matsuda and Cepko, 2004) and likely a small subset of postmitotic cells. The fate of electroporated cells in the mid-central retina was then analyzed at a mature time point (P21).

Cells expressing each NeuroD factor were identified by GFP expression and compared with control retinae electroporated with GFP alone. When possible, NeuroD protein expression was verified by antibody staining (supplemental Fig. 9, available at www.jneurosci.org as supplemental material). Cell fate was determined by position of the cell within the retina and expression of one or more molecular markers of retinal cell class. In electroporated retinae, photoreceptors were identified by their characteristic morphology and location in the ONL. In the INL, bipolar cells were identified by expression of Chx10, but not Sox9, p27, or Pax6. Müller glia were identified by expression of Sox9 or p27. Amacrine cells were identified by expression of Pax6, but not Sox9 or $\mathrm{p} 27$.

Among cells electroporated with GFP alone ( $n=7976$ cells, 3 retinae), the majority became rod photoreceptors in the ONL of electroporated retinae (Figs. $7 A-D, 8 A$ ). Smaller percentages of electroporated cells became bipolar, Müller glial or amacrine cells in the INL (Figs. $7 A-D, 8 B-D$ ). Of cells that were electroporated with NeuroD1 ( $n=9286$ cells, 3 retinae), a greater percentage adopted the photoreceptor cell fate when compared with control electroporations (Figs. $7 E-H, 8 A$ ). This came at the expense of Müller glia and possibly bipolar cell fates, whereas the percentage of cells adopting the amacrine cell fate was unchanged (Figs. $7 E-H, 8 B-D)$. By contrast, cells electroporated with NeuroD2 ( $n=4763$ cells, 3 retinae) demonstrated an approximately two- 


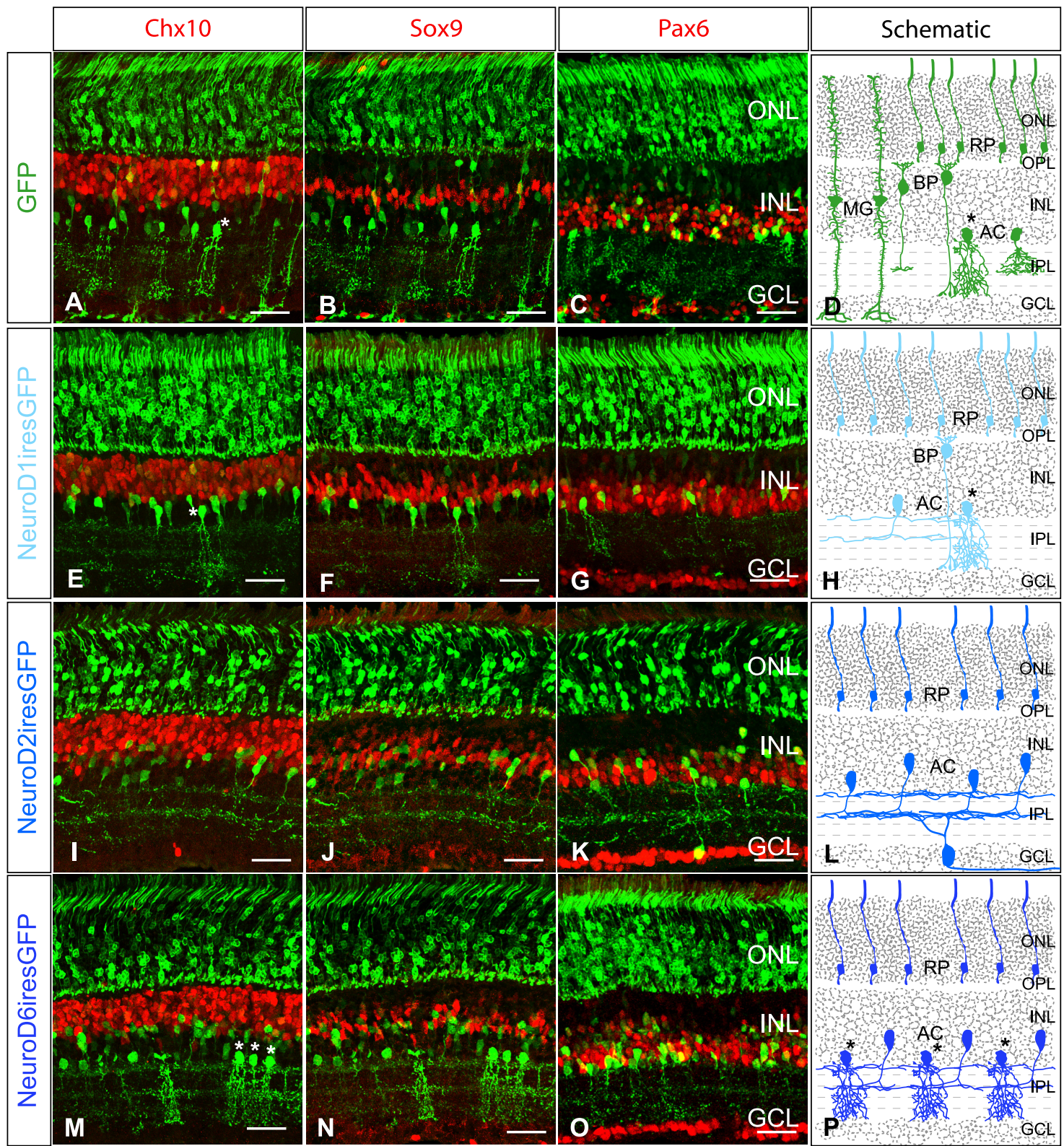

Figure 7. Forced expression of individual NeuroD family members in the newborn mouse retina. Plasmids encoding GFP, NeuroD1-ires-GFP, NeuroD2-ires-GFP, or NeuroD6-ires-GFP were individually electroporated in vivo into P0 mouse retinae. The fates of electroporated cells were then analyzed in the mature retina at P21. $A-D$, In vivo electroporation of GFP at P0 labeled photoreceptor cells in the $0 \mathrm{NL}$ of mature retinae, as well as $\mathrm{Chx} 10^{+}(\mathrm{red} ; \boldsymbol{A})$ bipolar cells, Sox9 ${ }^{+}(\mathrm{red} ; \boldsymbol{B})$ Müller glial cells, and Pax6 ${ }^{+}(\mathrm{red} ; \boldsymbol{C})$ amacrine cells in the INL. $\boldsymbol{E}-\boldsymbol{H}$, Electroporation of NeuroD1 gave rise to photoreceptors, Chx $10^{+}$bipolar cells, and Pax $6^{+}$amacrine cells, but suppressed the Sox $9^{+}$Müller glial cell fate. $I-L$, Cells electroporated with NeuroD2 became photoreceptors and Pax6 ${ }^{+}$amacrine cells, but did not adopt Chx $10^{+}$bipolar or Sox $9^{+}$Müller glial cell fates. $\boldsymbol{K}$, Cells electroporated with NeuroD2 were also observed in the GCL. $\boldsymbol{M}-\boldsymbol{P}$, Electroporation of NeuroD6 gave rise to photoreceptors and amacrine cells, but suppressed bipolar and Müller glial cells. Scale bar, $40 \mu \mathrm{m}$. The asterisk $\left(^{*}\right)$ denotes cells with All amacrine cell type-like morphology.

fold increase in the percentage of cells that adopted an amacrine cell fate and a respective decrease in bipolar cells and Müller glia (Figs. 7I-L, $8 B-D$ ). Photoreceptors were unchanged (Figs. 7I-L, $8 A$ ). Among cells electroporated with NeuroD6 ( $n=7732$ cells, 3 retinae), there was a greater than threefold increase in the percentage of cells adopting the amacrine cell fate compared with control cells, whereas the bipolar and Müller cell fates were suppressed (Figs. $7 M-P, 8 B-D$ ).

Each NeuroD family member altered the fate of electroporated cells in a manner different from control retinae. Comparison between individual NeuroD family members also revealed that each of these factors differed from one another, with respect to their effects on cell fate. For example, NeuroD2 induced significantly fewer bipolar cells ( $p=0.0018)$ and significantly more amacrine cells $(p=0.049)$ than NeuroD1. NeuroD6 also induced significantly fewer bipolar cells than NeuroD1 ( $p=$ $0.0014)$, significantly fewer photoreceptors $(p=0.00014)$, and significantly more amacrine cells $(p<0.0001)$. Finally, NeuroD6 induced significantly fewer photoreceptors $(p=0.0069)$ and significantly more amacrine cells than NeuroD2 $(p=0.016)$.

\section{Forced expression of NeuroD2 at P0 induces ganglion cell genesis}

Unexpectedly, a small percentage of cells in the central retina electroporated with NeuroD1, NeuroD2, or NeuroD6 were found in the GCL (Figs. $7 K, 8 E$ ). This was surprising because these cells are very rarely observed in control electroporations 

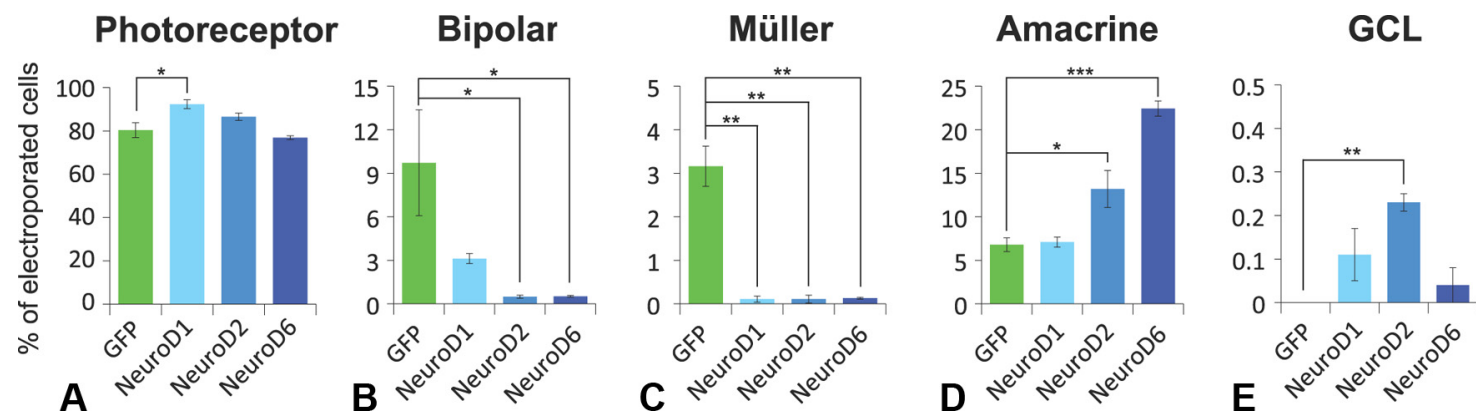

Figure 8. Quantitative analysis of cell fate in the mature retina after forced expression of individual NeuroD family members at P0.A-E, In vivo electroporation of GFP at PO labeled photoreceptors, bipolar cells, Müller glia, and amacrine cells. $\boldsymbol{A}, \boldsymbol{C}$, Electroporation of NeuroD1 gave rise to a small, but significant increase in photoreceptors and a significant decrease in Müller glial cells. $\boldsymbol{C}-\boldsymbol{E}$, Electroporation of NeuroD2 gave rise to significant increases in the percentage of amacrine cells and cells in the GCL, and a significant suppression of Müller glial cells. Electroporation of NeuroD6 gave rise to a significant increase in amacrine cells, compared with GFP alone, and a significant decrease in Müller glial cells. $n \geq 3$ retinae per condition; $>950$ cells were counted per retina. ${ }^{*} p<0.05$, ${ }^{* *} p<0.005,{ }^{* * *} p<0.0005$. Error bars indicate SEM.

and because most cells located in the GCL of the central retina are born before P0 (Young, 1985). Although the percentages of NeuroD-induced cells in the GCL are small, cells electroporated with NeuroD2 were significantly more prevalent in the GCL compared with control electroporations (Fig. 8). NeuroD2 also induced significantly more cells in the GCL compared with NeuroD6 $(p=0.015)$.

Based on their position in the GCL, these cells may be ganglion cells, displaced amacrine cells, or a mixed population of both classes of cells. To distinguish between these possibilities, we used Brn3, a broad marker for the ganglion cell class. Some of the NeuroD2-electroporated cells in the GCL expressed Brn3, whereas others did not, suggesting that these cells are a mixed population of ganglion and displaced amacrine cells (supplemental Fig. 10, available at www.jneurosci.org as supplemental material). To further verify that a subset of the cells in the GCL induced by NeuroD2 electroporation were ganglion cells, we analyzed the optic nerve head $(\mathrm{ONH})$ of retinae electroporated with NeuroD2-ires-GFP or control retinae. If a subset of cells in the GCL induced by NeuroD2 electroporation were ganglion cells, GFP-labeled axons would be visible in the ONH because the axons of ganglion cells leave the retina through this region to enter the optic nerve. Retinae were electroporated at P0 with NeuroD2ires-GFP or the control plasmid and harvested at P12, a time point when a greater percentage of cells electroporated with NeuroD2 were visible in the GCL (Fig. 9). In retinae electroporated with NeuroD2-ires-GFP, axons from labeled ganglion cells were clearly visible in the fiber layer and $\mathrm{ONH}$, whereas no GFPexpressing axons were visible in the fiber layer or $\mathrm{ONH}$ of control retinae (Fig. 9B,D). To follow the axons of these induced ganglion cells to specific retinorecipient areas of the brain, electroporated retinae were injected in vivo with the tracer CTb-594. Rare axons from NeuroD2-ires-GFP-expressing ganglion cells were found at the optic chiasm; however, no axons were found in the optic tract or in retinorecipient brain nuclei (data not shown). This may be the result of incomplete filling of induced ganglion cell axons with GFP. However, additional attempts to label the ganglion cell axons with an electroporated plasmid encoding the very sensitive reporter, alkaline phosphatase, along with GFP also failed to demonstrate NeuroD2-induced ganglion cell axons in the optic tract or retinorecipient regions of the brain. This suggests the possibility that, although ganglion cells induced by NeuroD2 are able to extend axons into the optic nerve, these axons may subsequently display pathfinding defects.

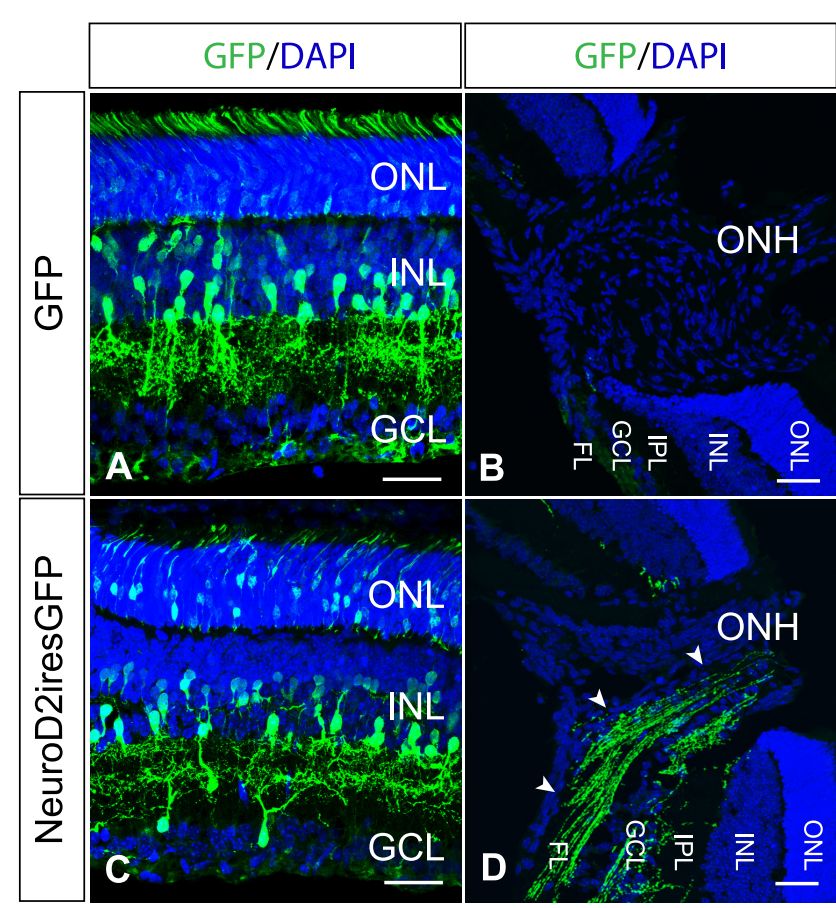

Figure 9. Forced expression of NeuroD2 in the newborn retina induces ganglion cell genesis Plasmids encoding GFP or NeuroD2-ires-GFP were individually electroporated in vivo into PO mouse retinae. Electroporated retinae were then analyzed at P12. $A$, Electroporation of GFP alone at P0 labels cells in the ONL and INL at P12. B, Cells electroporated with GFP at P0 do not extend axons into $0 \mathrm{NH}$ and GFP-expressing ganglion cell axons are not present in the fiber layer (FL) of the retina. $C, D$, Electroporation of NeuroD2 at PO results in cells in the $0 N L$, INL, and $G C L$ at P12. Axons from ganglion cells induced by forced expression of NeuroD2 are present in the fiber layer of the retina and in the $0 \mathrm{NH}$. Scale bar, $40 \mu \mathrm{m}$.

\section{Forced expression of individual NeuroD family members at} P3 had differential effects on neuronal cell fate and suppressed gliogenesis

The ability of retinal progenitor cells to give rise to different cell fates changes over developmental time. To establish whether the effects of NeuroD family member expression are also dependent on developmental time, we overexpressed each NeuroD family member individually at P3. Of cells electroporated at P3 with GFP alone ( $n=7948$ cells, 3 retinae), most adopted the rod photoreceptor, bipolar, or Müller glial cell fates (Figs. 10A-D, 11), consistent with the competency of retinal progenitor cells to produce these later-born cell classes (Young, 1985). Only a small percentage 


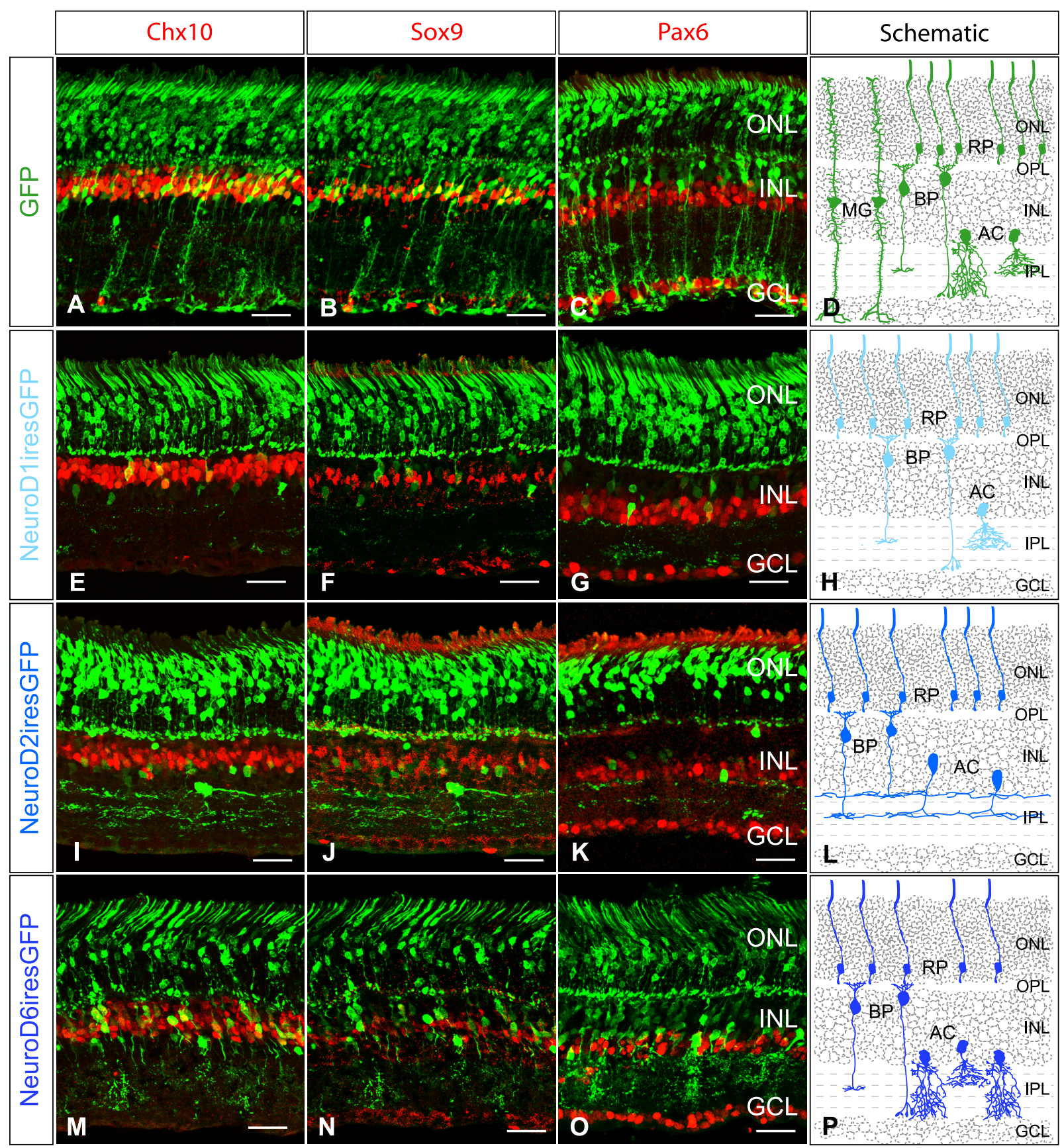

Figure 10. Forced expression of individual NeuroD family members in the mouse retina at P3. Plasmids encoding GFP, NeuroD1-ires-GFP, NeuroD2-ires-GFP, or NeuroD6-ires-GFP were individually electroporated in vivo into P3 mouse retinae. The fates of electroporated cells were then analyzed in the mature retina at P21. $\boldsymbol{A}-\boldsymbol{D}$, In vivo electroporation of GFP at P3 labeled photoreceptor cells in the $0 \mathrm{NL}$, as well as Chx10 ${ }^{+}$bipolar cells (red; $\boldsymbol{A}$ ), Sox9 ${ }^{+}$Müller glial cells (red; $\left.\boldsymbol{B}\right)$, and Pax6 ${ }^{+}$amacrine cells (red; $\boldsymbol{C}$ ), in the INL. $\boldsymbol{E}-\boldsymbol{P}$, Electroporation of NeuroD1 $(\boldsymbol{E}-\boldsymbol{H}$ ), NeuroD2 (I-L), or NeuroD6 $(\boldsymbol{M}-\boldsymbol{P})$ gave rise to photoreceptors, Chx10 ${ }^{+}$bipolar cells, and Pax $6^{+}$amacrine cells, but suppressed the Sox $9^{+}$Müller glial cell fate. Scale bar, $40 \mu \mathrm{m}$.

of cells adopted the amacrine cell fate. The fates of NeuroD1overexpressing cells ( $n=5802$ cells, 3 retinae) were not significantly different from control cells, except that the Müller glial cell fate was strongly suppressed (Figs. 10F, 11C). NeuroD2 and NeuroD6 electroporation similarly prevented cells from adopting the Müller glial cell fate (Figs. 10 J, N, 11C). In contrast to control and NeuroD1, however, significantly more cells electroporated with NeuroD2 $(n=$ 1739 cells, 3 retinae) or NeuroD6 ( $n=1648$ cells, 2 retinae) adopted the amacrine cell fate (Fig. $10 \mathrm{~K}, \mathrm{O}$ ). Similar to electroporation at P0, approximately twofold more cells electroporated with NeuroD2 at P3 became amacrine cells compared with control retinae (Fig. 11D). Threefold more cells electroporated with NeuroD6 at P3 became amacrine cells compared with control retinae. Electroporation of NeuroD6 also resulted in a small, but significant, reduction in the percentage of cells that became photoreceptors compared with control retinae (Fig. 11 A). In NeuroD2 electroporations, rare ganglion cells were observed; however, the ability of NeuroD2 to induce ganglion cells was significantly reduced when it was delivered at P3 compared with P0. No ganglion cells were labeled by electroporation with GFP alone at P0 or P3.

Comparing NeuroD family members directly with one another, significantly more NeuroD2 electroporated cells adopted an amacrine cell fate compared with NeuroD1 electroporated cells ( $p=$ 0.0036). Likewise, more NeuroD6-electroporated cells adopted an 
amacrine cell fate compared with NeuroD1electroporated cells ( $p=0.025$ ).

\section{NeuroD family members induced} neurite targeting to specific laminae of the inner plexiform layer

In addition to influencing cell fate, electroporation of NeuroD family members influenced targeting of the processes of amacrine cells to specific laminae within the IPL (Figs. 7, 12). At P0, the amacrine cell types labeled by in vivo electroporation of GFP are predominantly diffusely stratified GlyT1 ${ }^{+}$glycinergic cells, including the Dab1 ${ }^{+}$, AII amacrine cell type (Fig. 12 A, E, I,M) (Cherry et al., 2009). In retinae electroporated with NeuroD1 or NeuroD6, AII amacrine cells are also identifiable according to their distinctive morphology (Fig. $7 E, M$, white asterisks) and expression of Dab1 (Fig. $12 J-L$, white arrows). However, introduction of any of the NeuroD genes by electroporation at P0 appeared to bias other amacrine cell processes to two to three specific laminae in the mature IPL (Figs. $7 E-P ; 12 E-P$, white chevrons). These neurites intercalated between the processes of $\mathrm{ChAT}^{+}$cholinergic amacrine cell processes in the IPL (Fig. $12 \mathrm{E}-\mathrm{H}$, white chevrons). In contrast to the NeuroD1- and NeuroD6-electroporated retinae, no morphologically identifiable AII amacrine cells could be observed in retinae electroporated with NeuroD2 (Figs. 7I-K, $12 C, G, K, O)(n=10$ electroporated retinae). Electroporation of NeuroD2 led to a disappearance of morphologically normal AII amacrine cells; however, electroporated AII amacrine cells were still present in NeuroD2-electroporated retinae according to expression of Dab1 (Fig. $12 \mathrm{~K}, \mathrm{O}$, white arrowheads). Nonetheless, these cells no longer produced their stereotyped diffusely stratified morphology. Instead, their processes appeared to join with other amacrine cell neurites into two to three distinct laminae of the IPL (Fig. 12C,G, K,O).

\section{Discussion}

\section{Cell type-specific expression of}

bHLH factors

The bHLH family of transcription factors has been shown to have multiple roles in the developing CNS. Many bHLHs are expressed in progenitor cells and influence cell cycle exit, as well as cell fate (Sommer et al., 1996; Cepko, 1999; Bertrand et al., 2002; Guillemot, 2007; Ma et al., 2008). Expression of bHLHs is also in seen postmitotic precursor cells, as they are differentiating to adopt the characteristics of a particular cell fate, as well as in cells of the mature retina (Chien et al., 1996; Morrow

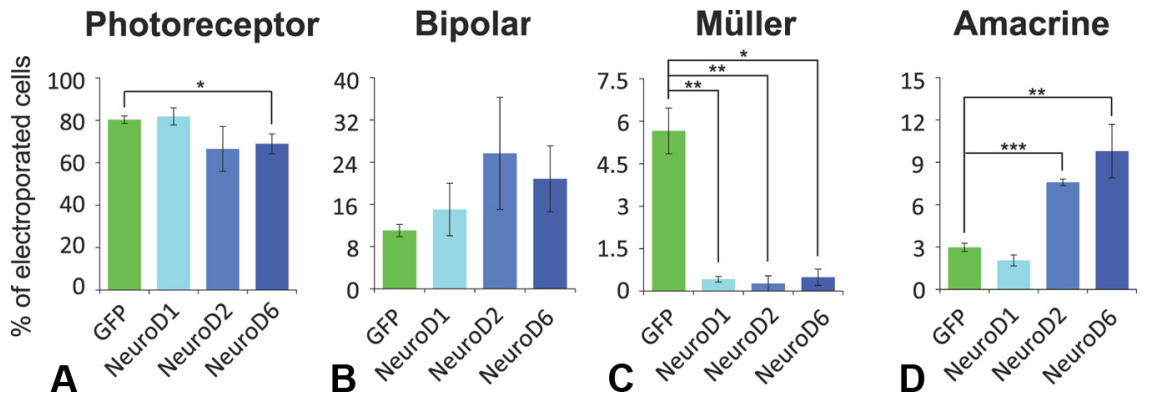

Figure 11. Quantitative analysis of cell fate in the mature retina after forced expression of individual NeuroD family members at P3. A-D, The greatest percentage of cells electroporated in vivo at P3 with GFP became photoreceptors, followed by bipolar cells, Müller glia, and amacrine cells. $\boldsymbol{C}$, Electroporation of NeuroD1 did not significantly change the percentages of cells becoming photoreceptor, bipolar, or amacrine cells, but resulted in a significant decrease in Müller glial cells. C, D, Electroporation of NeuroD2 gave rise to significant increases in the percentage of amacrine cell and a significant suppression of Müller glial cells. Electroporation of NeuroD6 gave rise to a significant increase in amacrine cells and significant decreases in photoreceptors and Müller glial cells. $n \geq 3$ retinae per condition, except NeuroD6, $n=2 ;>950$ cells were counted per retina. ${ }^{*} p<0.05,{ }^{* *} p<0.005,{ }^{* * *} p<$ 0.0005 . Error bars indicate SEM

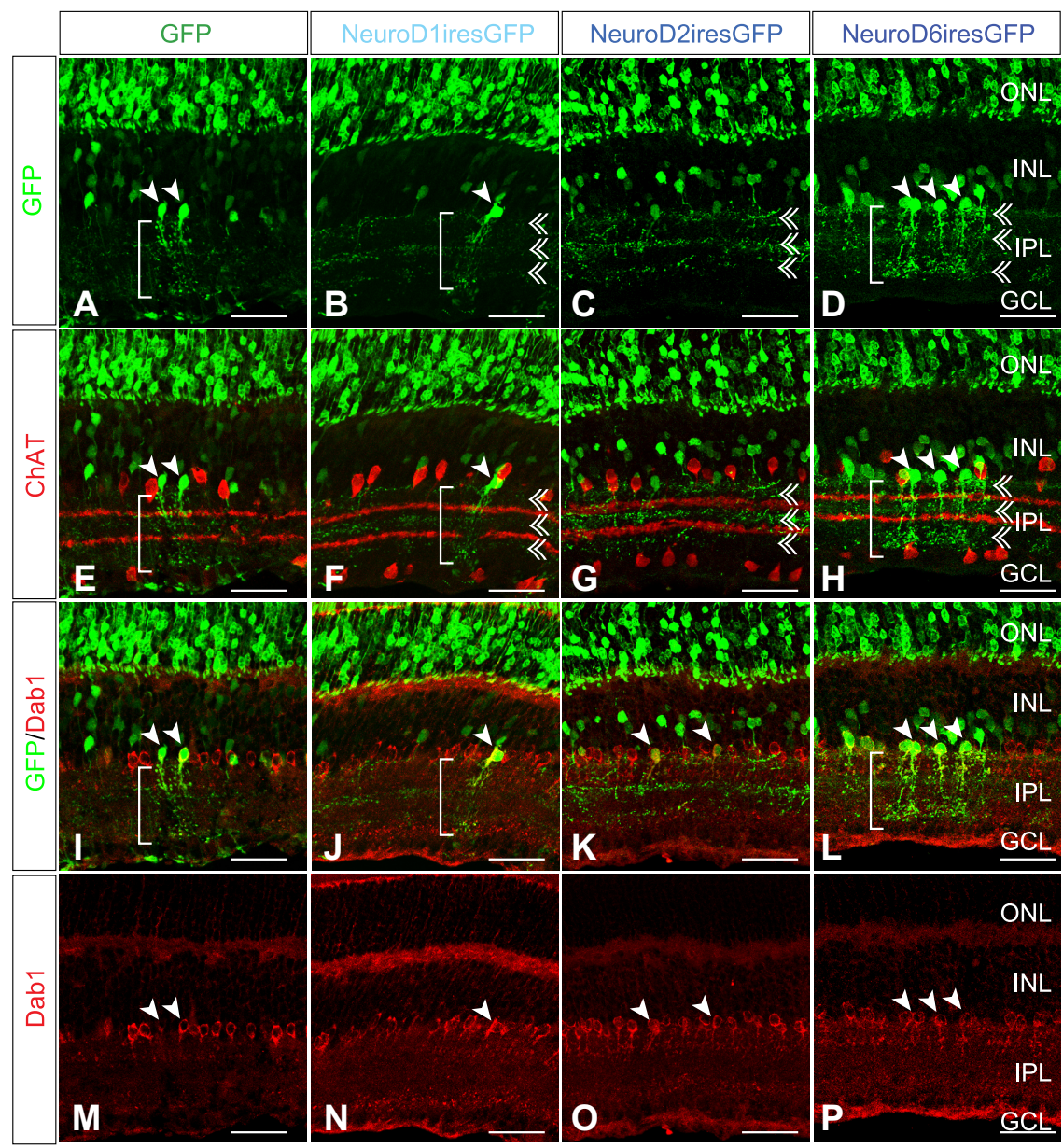

Figure 12. Stratification of neuronal processes in the IPL at $P 21$ after introduction of individual NeuroD factors in vivo at $P 0 . A$ Electroporation of the newborn mouse retina with GFP (green)-labeled amacrine cells in the lower INL that displayed diffusely stratified neuritic arbors in the IPL, including amacrine cells with stereotyped All amacrine cell morphology (white arrowhead, white brackets). $\boldsymbol{B}-\boldsymbol{D}$, Cells with stereotyped All amacrine cell morphologies were also found in NeuroD1 and NeuroD6 electroporated retinae, but not in retinae electroporated with NeuroD2. $\boldsymbol{A}-\boldsymbol{H}$, Compared with GFP alone, individual NeuroD factors bias the localization of certain neurites in the IPL to three distinct sublaminae (white chevrons), which intercalate between $\mathrm{ChAT}^{+}$ amacrine cell processes (red) $(\boldsymbol{E}-\boldsymbol{H}) . \boldsymbol{I}, \boldsymbol{J}, \boldsymbol{L}, \boldsymbol{M}, \boldsymbol{N}, \boldsymbol{P}$, Cells electroporated with GFP, NeuroD1, or NeuroD6 have characteristic All amacrine cell morphology and colocalize with Dab1, a molecular marker for All amacrine cells (white arrowheads). $\boldsymbol{K}, \mathbf{0}$, Cells electroporated with NeuroD2 colocalize with Dab1, but do not possess typical All amacrine cell morphology and instead costratify with other processes. Scale bars, $40 \mu \mathrm{m}$. 


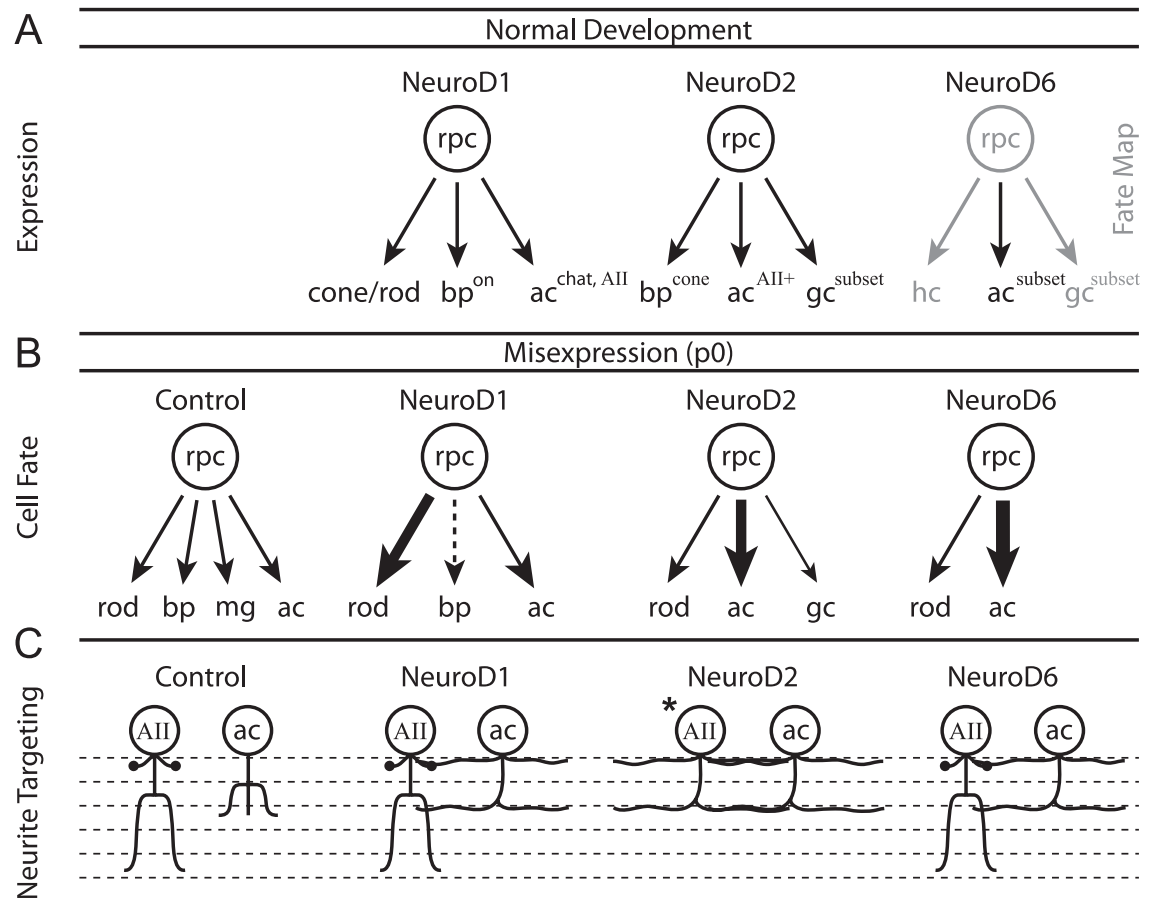

Figure 13. Summary of NeuroD family member expression and gain-of-function analyses in the murine retina. $\boldsymbol{A}$, NeuroD1 is expressed in a subset of retinal progenitor cells $(r p c)$ in the developing retina. In the mature retina, NeuroD 1 is expressed in rods and cones, on-bipolar cells $\left(\mathrm{bp}^{\mathrm{on}}\right)$, and in cholinergic and All amacrine cells ( $\left.\mathrm{ac}^{\text {chat,All }}\right)$. NeuroD2 is expressed in a smaller subset of retinal progenitor cells during development and in subsets of cone-bipolar (bp ${ }^{\text {cone }}$ ) and ganglion ( $g c$ c) cells. NeuroD2 is also expressed in All amacrine cells and another subset of amacrine cells $\left(\mathrm{ac}^{\mathrm{All}+}{ }^{+}\right)$. NeuroD6 is expressed in non-GABAergic, nonglycinergic amacrine cells ( $\left(\mathrm{c}^{\text {subset }}\right.$ ) in the adult retina. Horizontal cells ( $\mathrm{hc}$ ) and a subset of ganglion cells ( $\mathrm{g} \mathrm{c}^{\text {subset}}$ ) have a history of NeuroD6 expression, but this expression is not maintained in the adult. It is unknown whether rpcs expressing one or more NeuroD family members give rise to the cell types that express NeuroD genes in the mature retina. $\boldsymbol{B}$, The effects of NeuroD family member misexpression on retinal cell fate at $\mathrm{P} 0$. The relative thickness of arrows reflects the production of each cell class compared with electroporation of GFP alone. Electroporation of the retina with GFP at PO labels rpc and postmitotic cells that give rise to rod photoreceptors (rod), bipolar cells (bp), Müller glia (mg), and amacrine cells. Misexpression of NeuroD1 by electroporation increases the percentage of cells that adopt the rod photoreceptor cell fate, reduces the percentage of bipolar cells produced, and suppresses the Müller glial cell fate, but does not affect amacrine cell production. Misexpression of NeuroD2 suppresses the Müller glial and bipolar cell fates and induces amacrine cell and ganglion cell production, but does not influence the rod photoreceptor cell fate. NeuroD6 expression induces amacrine cells most strongly, while suppressing bipolar and Müller glial cell production. The photoreceptor fate is unchanged by NeuroD6 expression at PO. C, The effect of NeuroD family member expression on amacrine cell neurite targeting. Electroporation of GFP at P0 mostly labels diffusely stratified narrow-field amacrine cells. NeuroD1 and NeuroD6 do not affect All amacrine cell morphology; however, some bias is seen in other amacrine cell processes toward two to three specific strata in the IPL. NeuroD2 causes all amacrine cells, including All amacrine cells $\left(^{*}\right)$, to stratify to two to three specific strata within the IPL.

et al., 1999; Inoue et al., 2002; Ma et al., 2008). In the present study, we demonstrate that bHLHs are expressed in distinct, but overlapping sets of retinal cell types. These expression patterns suggest that bHLH factors may have a combinatorial role in the development of retinal cell diversity, not simply at the level of cell class, but also at the level of distinct cell types.

\section{The role of NeuroD factors in retinal cell fate}

The role of NeuroD1 in retinal development has been well characterized (Morrow et al., 1999; Inoue et al., 2002). Roles for NeuroD2 and NeuroD6 in the retina, however, have not been previously described. Elsewhere in the nervous system, these NeuroD factors are important for terminal differentiation, neuronal survival, and synaptogenesis (Olson et al., 2001; Ince-Dunn et al., 2006; Yang et al., 2009). In the retina, we found that each NeuroD family member appears to have a distinct role in effecting cell fate (Fig. 13). Loss of NeuroD2 in the retina resulted in a modest reduction in AII amacrine cells. Misexpression of any NeuroD factor was sufficient to suppress cells from adopting the Müller glial cell fate, in keeping with our previous observations of proneural bHLHs (Cai et al., 2000). However, the effects of each NeuroD factor differed with respect to the neuronal cell types it induced or suppressed (Fig. 13B). At P0, NeuroD1 potentiated photoreceptor production, whereas NeuroD2 and NeuroD6 induced amacrine cell production. Interestingly, NeuroD2 misexpression was able to induce ganglion cell production in the central retina, outside of the normal developmental window of ganglion cell birth (Young, 1985). At P3, as development progresses and fewer amacrine cells are normally being made in the retina, NeuroD2 and NeuroD6 were still able to potentiate amacrine cell production. However, these two factors no longer suppressed bipolar cell production, as they did at $\mathrm{P} 0$. This difference most likely reflects the changing competence of progenitor cells to produce different cell types over time, which resulted in different responses to the introduction of these genes.

The effects of NeuroD2 and NeuroD6 misexpression in the retina are distinct from previously studied bHLH factors in several ways. First, unlike previous studies in which single bHLH factors have been misexpressed in the developing retina, neither NeuroD2 nor NeuroD6 induced photoreceptor production at the expense of Müller glia (Morrow et al., 1999; Hatakeyama et al., 2001; Inoue et al., 2002). This demonstrates that these factors did not simply drive cells from the cell cycle and induce them to adopt the fate of the most abundantly made neuron type at the time that it was misexpressed. Another unusual aspect of NeuroD2 and NeuroD6 is that coexpression with a homeodomain transcription factor was not necessary to influence cell fate in a significant manner. Previous studies of Mash1 and Math3/NeuroD4 demonstrated that coexpression of either with Chx10 induced bipolar cells, whereas expression of the bHLHs singly induced only photoreceptor production (Hatakeyama et al., 2001). It is possible, however, that coexpression of NeuroD2 and NeuroD6 with a homeodomain could further potentiate or otherwise alter their effects on cell fate. A third remarkable effect of NeuroD2 misexpression was the induction of ganglion cells, a result that has not been reported previously for bHLH misexpression in the postnatal mouse retina. This observation demonstrates that NeuroD2 is sufficient to overcome some aspects of temporal progenitor cell patterning as ganglion cells were induced to form outside of their normal developmental window. Together, these observations suggest that the normal function of NeuroD genes may be to influence cell fate decisions in differentiating retinal cells, and to act further downstream in cell type-specific development to drive cell type-specific subprograms of gene expression. 


\section{NeuroD factors and retinal circuitry}

The expression of NeuroD1 in rod photoreceptors, rod bipolar cells, and AII amacrine cells outlines an important retinal circuit for vision under low light conditions (Fig. 13A). It may prove to be a coincidence that cells belonging to a specific neural circuit express the same bHLH family member. Indeed, other bipolar cell types, namely cone bipolar cell types, seem to express NeuroD1. Interestingly, all NeuroD1-expressing bipolar cells appear to stratify to the ON layer of the IPL and AII amacrine cells are known to be presynaptic to cone bipolar cells in this region. Indeed, this synapse forms the penultimate output of the rod circuit in the retina under low light conditions and underlies important visual functions (Bloomfield and Dacheux, 2001; Münch et al., 2009). Despite these correlations, cholinergic amacrine cells, which also express NeuroD1, are not currently known to play an essential part of the rod circuit. Among cells that express NeuroD2, only AII amacrine cells have a known morphology. Even less is known about the NeuroD6 ${ }^{+}$amacrine cells that do not express either GlyT1 or Gad1. To better characterize and investigate the circuitry of NeuroD-expressing cells, one could produce GFP knock-in lines capable of labeling the entire morphology of NeuroD ${ }^{+}$cells or construct a NeuroD promoter-reporter construct for electroporation. As these reagents become available, it may soon be possible to determine whether expression of each NeuroD factor is shared among specific synaptic partners and more importantly whether bHLHs function to define specific neural circuits.

The ability of NeuroD genes to target processes of electroporated cells to two to three specific laminae of the IPL suggests that a common transcriptional target of NeuroD factors may be involved in neurite pathfinding or homophilic adhesion (Fig. 13C). In the former case, the target(s) may instruct neuronal processes to actively seek these layers. In the latter case, homophilic adhesion among neurites of NeuroD-expressing neurons may cause outgrowing neurites to colocalize to the targets of the first pioneering processes. Alternatively, these laminae may be the only layers available for occupancy of neurites produced at this late time point. This is unlikely, however, because the majority of amacrine cells produced at $\mathrm{P} 0$ are diffusely stratifying glycinergic cells (Cherry et al., 2009). One intriguing detail is that NeuroD2 was the only NeuroD family member capable of influencing AII amacrine cell morphology. Although it is unclear why this may be, one possible explanation comes from the endogenous expression of NeuroD2 in AII amacrine cells. It may be that AII amacrine cells normally express NeuroD2 and therefore are intrinsically sensitive to varying levels the transcription factor. Although preliminary, the finding that NeuroD factors influence neurite targeting in the IPL may suggest a functional link between bHLH expression and the formation of neural circuits among molecularly defined neuronal cell types.

\section{Conclusion}

The specification and differentiation of a distinct neuronal cell type is a complex process. A cell must leave the cell cycle, migrate to an appropriate position, find the correct synaptic partners, and express the appropriate battery of genes necessary for its distinct function. This complex process can be simplified by considering it as a collection of individual fate decisions integrated over time (Jukam and Desplan, 2010). Transcription factors undoubtedly play important roles in orchestrating these individual decisions to arrive at a distinct fate (Cepko, 1999). In one model, a transcription factor may act as a terminal selector of cell fate by directly controlling expression of a battery of terminal differ- entiation genes through common cis-regulatory target sites (Hobert, 2008). Alternatively, a transcription factor may act downstream of such terminal selector genes as part of a feedforward loop driving one particular aspect of cell fate (Hobert, 2008; Baumgardt et al., 2009). Last, a single transcription factor may act in a combinatorial fashion with other transcription factors to produce a large diversity of cell fates while minimizing the number of necessary factors (Guillemot, 2007). Cell type-specific expression of NeuroD factors may represent this third model. On one hand, NeuroD factors are capable of driving cells out of the cell cycle, selecting neuronal over glial cell fates, and directing cells to adopt a particular class identity. In the case of NeuroD2, this influence over cell fate can even occur outside the normal window of development for a particular cell class. This alone might suggest that NeuroD2 functions as a terminal selector gene; however, its expression is not restricted to a single cell type. Moreover, cell fates induced by NeuroD2 misexpression display only parts of the normal morphological development that is characteristic of these cells. Because NeuroD genes act in the early stages of fate specification as well as the later stages of morphological development, a multifaceted and combinatorial role for these transcriptional factors is most likely.

\section{References}

Baumgardt M, Karlsson D, Terriente J, Díaz-Benjumea FJ, Thor S (2009) Neuronal subtype specification within a lineage by opposing temporal feed-forward loops. Cell 139:969-982.

Bertrand N, Castro DS, Guillemot F (2002) Proneural genes and the specification of neural cell types. Nat Rev Neurosci 3:517-530.

Blackshaw S, Harpavat S, Trimarchi J, Cai L, Huang H, Kuo WP, Weber G, Lee K, Fraioli RE, Cho SH, Yung R, Asch E, Ohno-Machado L, Wong WH, Cepko CL (2004) Genomic analysis of mouse retinal development. PLoS Biol 2:E247.

Bloomfield SA, Dacheux RF (2001) Rod vision: pathways and processing in the mammalian retina. Prog Retin Eye Res 20:351-384.

Bramblett DE, Pennesi ME, Wu SM, Tsai MJ (2004) The transcription factor Bhlhb4 is required for rod bipolar cell maturation. Neuron 43:779-793.

Cai L, Morrow EM, Cepko CL (2000) Misexpression of basic helix-loophelix genes in the murine cerebral cortex affects cell fate choices and neuronal survival. Development 127:3021-3030.

Cepko CL (1999) The roles of intrinsic and extrinsic cues and bHLH genes in the determination of retinal cell fates. Curr Opin Neurobiol 9:37-46.

Cherry TJ, Trimarchi JM, Stadler MB, Cepko CL (2009) Development and diversification of retinal amacrine interneurons at single cell resolution. Proc Natl Acad Sci U S A 106:9495-9500.

Chien CT, Hsiao CD, Jan LY, Jan YN (1996) Neuronal type information encoded in the basic-helix-loop-helix domain of proneural genes. Proc Natl Acad Sci U S A 93:13239-13244.

Feng L, Xie X, Joshi PS, Yang Z, Shibasaki K, Chow RL, Gan L (2006) Requirement for Bhlhb5 in the specification of amacrine and cone bipolar subtypes in mouse retina. Development 133:4815-4825.

Fuerst PG, Koizumi A, Masland RH, Burgess RW (2008) Neurite arborization and mosaic spacing in the mouse retina require DSCAM. Nature 451:470-474.

Fuerst PG, Bruce F, Tian M, Wei W, Elstrott J, Feller MB, Erskine L, Singer JH, Burgess RW (2009) DSCAM and DSCAML1 function in self-avoidance in multiple cell types in the developing mouse retina. Neuron 64:484-497.

Gaudillière B, Konishi Y, de la Iglesia N, Yao G, Bonni A (2004) A CaMKIINeuroD signaling pathway specifies dendritic morphogenesis. Neuron 41:229-241.

Goebbels S, Bormuth I, Bode U, Hermanson O, Schwab MH, Nave KA (2006) Genetic targeting of principal neurons in neocortex and hippocampus of NEX-Cre mice. Genesis 44:611-621.

Gradwohl G, Fode C, Guillemot F (1996) Restricted expression of a novel murine atonal-related bHLH protein in undifferentiated neural precursors. Dev Biol 180:227-241. 
Guillemot F (2007) Spatial and temporal specification of neural fates by transcription factor codes. Development 134:3771-3780.

Hatakeyama J, Kageyama R (2004) Retinal cell fate determination and bHLH factors. Semin Cell Dev Biol 15:83-89.

Hatakeyama J, Tomita K, Inoue T, Kageyama R (2001) Roles of homeobox and bHLH genes in specification of a retinal cell type. Development 128:1313-1322.

Hobert O (2008) Regulatory logic of neuronal diversity: terminal selector genes and selector motifs. Proc Natl Acad Sci U S A 105:20067-20071.

Ince-Dunn G, Hall BJ, Hu SC, Ripley B, Huganir RL, Olson JM, Tapscott SJ, Ghosh A (2006) Regulation of thalamocortical patterning and synaptic maturation by NeuroD2. Neuron 49:683-695.

Inoue T, Hojo M, Bessho Y, Tano Y, Lee JE, Kageyama R (2002) Math3 and NeuroD regulate amacrine cell fate specification in the retina. Development 129:831-842.

Jukam D, Desplan C (2010) Binary fate decisions in differentiating neurons. Curr Opin Neurobiol 20:6-13.

Kim DS, Ross SE, Trimarchi JM, Aach J, Greenberg ME, Cepko CL (2008) Identification of molecular markers of bipolar cells in the murine retina. J Comp Neurol 507:1795-1810.

Ledent V, Paquet O, Vervoort M (2002) Phylogenetic analysis of the human basic helix-loop-helix proteins. Genome Biol 3:RESEARCH0030.

Lee JE, Hollenberg SM, Snider L, Turner DL, Lipnick N, Weintraub H (1995) Conversion of Xenopus ectoderm into neurons by NeuroD, a basic helixloop-helix protein. Science 268:836-844.

Ma YC, Song MR, Park JP, Henry Ho HY, Hu L, Kurtev MV, Zieg J, Ma Q, Pfaff SL, Greenberg ME (2008) Regulation of motor neuron specification by phosphorylation of neurogenin 2 . Neuron 58:65-77.

Masland RH (2001) Neuronal diversity in the retina. Curr Opin Neurobiol 11:431-436.

Masland RH (2004) Neuronal cell types. Curr Biol 14:R497-R500.

Matsuda T, Cepko CL (2004) Electroporation and RNA interference in the rodent retina in vivo and in vitro. Proc Natl Acad Sci U S A 101:16-22.

Matsuoka RL, Nguyen-Ba-Charvet KT, Parray A, Badea TC, Chédotal A, Kolodkin AL (2011) Transmembrane semaphorin signalling controls laminar stratification in the mammalian retina. Nature 470:259-263.

Miyata T, Maeda T, Lee JE (1999) NeuroD is required for differentiation of the granule cells in the cerebellum and hippocampus. Genes Dev 13:1647-1652.

Morrow EM, Furukawa T, Lee JE, Cepko CL (1999) NeuroD regulates mul- tiple functions in the developing neural retina in rodent. Development 126:23-36.

Münch TA, da Silveira RA, Siegert S, Viney TJ, Awatramani GB, Roska B (2009) Approach sensitivity in the retina processed by a multifunctional neural circuit. Nat Neurosci 12:1308-1316.

Olson JM, Asakura A, Snider L, Hawkes R, Strand A, Stoeck J, Hallahan A, Pritchard J, Tapscott SJ (2001) NeuroD2 is necessary for development and survival of central nervous system neurons. Dev Biol 234:174-187.

Roesch K, Jadhav AP, Trimarchi JM, Stadler MB, Roska B, Sun BB, Cepko CL (2008) The transcriptome of retinal Muller glial cells. J Comp Neurol 509:225-238.

Salic A, Mitchison TJ (2008) A chemical method for fast and sensitive detection of DNA synthesis in vivo. Proc Natl Acad Sci U S A 105:2415-2420.

Sanes JR, Zipursky SL (2010) Design principles of insect and vertebrate visual systems. Neuron 66:15-36.

Schwab MH, Bartholomae A, Heimrich B, Feldmeyer D, Druffel-Augustin S, Goebbels S, Naya FJ, Zhao S, Frotscher M, Tsai MJ, Nave KA (2000) Neuronal basic helix-loop-helix proteins (NEX and BETA2/Neuro D) regulate terminal granule cell differentiation in the hippocampus. J Neurosci 20:3714-3724.

Sommer L, Ma Q, Anderson DJ (1996) neurogenins, a novel family of atonal-related bHLH transcription factors, are putative mammalian neuronal determination genes that reveal progenitor cell heterogeneity in the developing CNS and PNS. Mol Cell Neurosci 8:221-241.

Soriano P (1999) Generalized lacZ expression with the ROSA26 Cre reporter strain. Nat Genet 21:70-71.

Trimarchi JM, Stadler MB, Roska B, Billings N, Sun B, Bartch B, Cepko CL (2007) Molecular heterogeneity of developing retinal ganglion and amacrine cells revealed through single cell gene expression profiling. J Comp Neurol 502:1047-1065.

Trimarchi JM, Stadler MB, Cepko CL (2008) Individual retinal progenitor cells display extensive heterogeneity of gene expression. PLoS One 3:e1588.

Yamagata M, Weiner JA, Sanes JR (2002) Sidekicks: synaptic adhesion molecules that promote lamina-specific connectivity in the retina. Cell 110:649-660.

Yang Y, Kim AH, Yamada T, Wu B, Bilimoria PM, Ikeuchi Y, de la Iglesia N, Shen J, Bonni A (2009) A Cdc20-APC ubiquitin signaling pathway regulates presynaptic differentiation. Science 326:575-578.

Young RW (1985) Cell differentiation in the retina of the mouse. Anat Rec 212:199-205. 